\title{
On the arithmetic properties of complex values of Hecke-Mahler series I. The rank one case
}

\author{
FEDERICO PELLARIN
}

\begin{abstract}
Here we characterise, in a complete and explicit way, the relations of algebraic dependence over $\mathbb{Q}$ of complex values of Hecke-Mahler series taken at algebraic points $\underline{u}_{1}, \ldots, \underline{u}_{m}$ of the multiplicative group $\mathbb{G}_{\mathrm{m}}^{2}(\mathbb{C})$, under a technical hypothesis that a certain sub-module of $\mathbb{G}_{\mathrm{m}}^{2}(\mathbb{C})$ generated by the $\underline{u}_{i}$ 's has rank one (rank one hypothesis). This is the first part of a work, announced in [Pel1], whose main objective is completely to solve a general problem on the algebraic independence of values of these series.
\end{abstract}

Mathematics Subject Classification (2000): 11J85 (primary); 11J91 (secondary).

\section{Introduction, results}

Let $w$ be a real irrational positive number. The Hecke-Mahler series associated to $w$ is the power series:

$$
f_{w}(u, v)=\sum_{l=1}^{\infty} \sum_{h=1}^{[l w]} u^{l} v^{h}
$$

where the square brackets denote the greatest integer part. ${ }^{1}$ On the domain

$$
\mathcal{D}=\left\{(u, v) \in \mathbb{C}^{2} \text { with }|u|<1 \text { and }|u||v|^{w}<1\right\},
$$

this series converges to a function which is transcendental over $\mathbb{C}(u, v)$ (cf. [Ni, page 43]). Throughout this text, convergence means absolute convergence.

Let us consider an $m$-tuple of couples of non-zero complex numbers

$$
\mathcal{M}=\left(\left(u_{1}, v_{1}\right), \ldots,\left(u_{m}, v_{m}\right)\right)=\left(\underline{u}_{1}, \ldots, \underline{u}_{m}\right)
$$

such that $\left|u_{i}\right|<1$ and $\left|u_{i}\right|\left|v_{i}\right|^{w}<1(1 \leq i \leq m)$.

${ }^{1}$ If $l w<1$, we adopt the convention that the sum $\sum_{h=1}^{[l w]} u^{l} v^{h}$ is zero. Throughout this text, if a sum or a series has the empty set $\emptyset$ as set of indices, by convention it is equal to zero.

Received January 21, 2005; accepted in revised form July 7, 2006. 
We study the algebraic dependence relations over $\mathbb{Q}$ of the numbers

$$
f_{w}\left(\underline{u}_{1}\right), \ldots, f_{w}\left(\underline{u}_{m}\right)
$$

in the case of $\underline{u}_{i}$ algebraic over $\mathbb{Q}$ for all $i=1, \ldots, m$, and $w$ quadratic irrational. In all the following, we write $K=\mathbb{Q}(w)$.

In this text we also work under a condition over $\mathcal{M}$ that we call rank one hypothesis, depending on the choice of $w$ (in [Pel2] we consider the general case); this hypothesis will be formulated when $w=\theta$ satisfies:

$$
0<\theta<1 \text { and } \theta^{\prime}<-1,
$$

where $\alpha \mapsto \alpha^{\prime}$ is the non-trivial automorphism of $K$.

The stabiliser $S(M) \subset K$ of the module $M=\mathbb{Z}+\theta^{-1} \mathbb{Z}$ acts on the algebraic group $\mathbb{G}_{m}^{2}$ by $\underline{u} \mapsto \underline{u}^{\beta}$, where $\underline{u}=(u, v), \beta \in S(M)$, and $\underline{u}^{\beta}=\left(u^{a} v^{b}, u^{c} v^{d}\right)$ for the rational integers $\bar{a}, b, c, d$ defined by

$$
\beta \cdot \theta^{-1}=a \theta^{-1}+b, \quad \beta \cdot 1=c \theta^{-1}+d .
$$

The rank one hypothesis on $\underline{u}_{1}, \ldots, \underline{u}_{m}$ amounts to the existence of $\underline{v} \in \mathbb{G}_{m}^{2}$ and torsion points $\underline{\zeta}_{1}, \ldots, \underline{\zeta}_{m} \in \mathbb{G}_{m}^{2}$, together with non-zero elements $\beta_{1}, \ldots, \beta_{m} \in$ $S(M)$ such that

$$
\underline{u}_{i}=\left(u_{i}, v_{i}\right)=\underline{\zeta}_{i} \underline{v}^{\beta_{i}}, \quad 1 \leq i \leq m
$$

with respect to the group law on $\mathbb{G}_{m}^{2}$.

We denote by $\mathbb{C}\{U, V\}$ the set of formal double Laurent series

$$
\sum_{(l, h) \in \mathbb{Z}^{2}} c_{l, h} U^{l} V^{h}
$$

with $c_{l, h} \in \mathbb{C}$. Here, the sum $\sum_{(l, h) \in \mathbb{Z}^{2}} c_{l, h} U^{l} V^{h}$ is viewed as a notation for the map $c: \mathbb{Z}^{2} \rightarrow \mathbb{C}$ with $c(l, h)=c_{l, h}$. The set $\mathbb{C}\{U, V\}$ is a $\mathbb{C}$-vector space, hence a $\mathbb{Q}$-vector space.

Taking into account all these tools, notations and hypotheses (see Section 2 for more details), we prove the following result.

Theorem 1.1. Let $\underline{u}_{1}, \ldots, \underline{u}_{m}$ be algebraic points of $\mathbb{G}_{m}^{2} \cap \mathcal{D}$ and let us suppose that the $m$-tuple $\mathcal{M}=\left(\underline{u}_{1}, \ldots, \underline{u}_{m}\right)$ satisfies the rank one hypothesis, so that relations like (1.4) hold. The algebraic independence over $\mathbb{Q}$ of the complex numbers $f_{\theta}\left(\underline{u}_{1}\right), \ldots, f_{\theta}\left(\underline{u}_{m}\right)$ is equivalent to the $\mathbb{Q}$-linear independence of the series

$$
\sum_{(l, h) \in \mathbb{Z}^{2} \backslash\{(0,0)\}} U_{i}^{l} V_{i}^{h} \in \mathbb{C}\{U, V\}, \quad 1 \leq i \leq m
$$

where $\left(U_{i}, V_{i}\right)=\underline{\zeta}_{i} \underline{U}^{\beta_{i}}(1 \leq i \leq m)$. 
Moreover, if $f_{\theta}\left(\underline{u}_{1}\right), \ldots, f_{\theta}\left(\underline{u}_{m}\right)$ are algebraically dependent over $\mathbb{Q}$, then there is a non-trivial linear relation

$$
\sum_{i=1}^{m} c_{i} f_{\theta}\left(\underline{u}_{i}\right)=\lambda
$$

where $\lambda$ is an algebraic number and $c_{1}, \ldots, c_{m}$ are rational numbers.

Remark 1.2. Let $\mathcal{B}=\left(\begin{array}{ll}a & b \\ c & d\end{array}\right)$ be a matrix with rational integer entries $a, b, c, d$, let $\underline{u}=(u, v)$ be an element of $\mathbb{G}_{m}^{2}$. A useful notation that will be adopted throughout this text, is:

$$
\mathcal{B} \cdot \underline{u}=\left(u^{a} v^{b}, u^{c} v^{d}\right) .
$$

Thus, if $\beta \in S(M)$, then $\underline{u}^{\beta}=\mathcal{B} . \underline{u}$ where $a, b, c, d$ are defined by (1.3); in this case, we also write $\mathcal{B}=\mathcal{B}(\beta)$. This is the classical left action by integral matrices in Mahler's method, but here $S(M)$ is commutative so that, for all $\beta, \gamma \in S(M)$, $\left(\underline{u}^{\beta}\right)^{\gamma}=\left(\underline{u}^{\gamma}\right)^{\beta}=\underline{u}^{\beta \gamma}$.

Condition (1.2) means that $\theta^{-1}$ is reduced in the classical sense (cf. definition page 79 of [Per]). After [Per, Theorem 4, page 80], (1.2) holds if and only if $\theta^{-1}$ has the purely periodic continued fraction development:

$$
\theta^{-1}=\frac{1}{b_{1}+} \frac{1}{b_{2}+\cdots}=[\underbrace{b_{1}, b_{2}, \ldots, b_{2 r}}_{\text {period }}, b_{1}, b_{2}, \ldots]
$$

with $b_{1}, b_{2} \ldots \in \mathbb{Z}_{>0}$.

Condition (1.2) over $w=\theta$ can be dropped in our theorem in some cases. For the sake of simplicity, we assume that $0<w<1$; it has a continued fraction development

$$
w=\frac{1}{d_{1}+} \frac{1}{d_{2}+\cdots} \frac{1}{b_{1}+} \frac{1}{b_{2}+\cdots}=[0, d_{1}, d_{2} \ldots, d_{g}, \underbrace{b_{1}, b_{2}, \ldots, b_{2 r}}_{\text {period }}, b_{1}, b_{2}, \ldots]
$$

for $g \geq 0, r>0$ and $d_{1}, \ldots, d_{g}, b_{1}, \ldots, b_{2 r} \in \mathbb{Z}_{>0}$, with the convention that if $g=0$, then $w=\left[0, b_{1}, \ldots, b_{2 r}, \ldots\right]$.

Following [Ni, pages 43-44] or [Mas2, pages 210-211], we put:

$$
T=\left(\begin{array}{cc}
d_{g} & 1 \\
1 & 0
\end{array}\right) \cdots\left(\begin{array}{ll}
d_{1} & 1 \\
1 & 0
\end{array}\right)=\left(\begin{array}{ll}
a & b \\
c & d
\end{array}\right)
$$

when $g>0$, or $T=$ identity matrix when $g=0$ (that is, when the continued fraction development of $w^{-1}$ is purely periodic). Let $\theta \in \mathbb{Q}(w)$ be defined by $w=\frac{d \theta+b}{c \theta+a}$ : 
we note that $\theta^{-1}$ has the purely periodic continued fraction development (1.6). Moreover, we have:

$$
f_{w}(\underline{u})=f_{\theta}(T \cdot \underline{u})+\tilde{R}(\underline{u})
$$

for some rational function $\tilde{R} \in \mathbb{Q}(\underline{u})$ defined over couples of complex numbers $(u, v)$ such that $|u|<1$ and $|u||v|^{w}<1$ (cf. [Ni, page 44] and [Mas2, page 211, equation (3.6)]).

Hence, the complex numbers

$$
f_{w}\left(\underline{u}_{1}\right), \ldots, f_{w}\left(\underline{u}_{m}\right)
$$

are algebraically independent over $\mathbb{Q}$ if and only if the complex numbers

$$
f_{\theta}\left(T . \underline{u}_{1}\right), \ldots, f_{\theta}\left(T . \underline{u}_{m}\right)
$$

are algebraically independent over $\mathbb{Q}$. The algebraic dependence and the relations among the numbers (1.1) can be checked by using our theorem, provided that the $m$-tuple $\left(T . \underline{u}_{1}, \ldots, T . \underline{u}_{m}\right)$ satisfies the rank one hypothesis ("associated" to $\theta$ ).

It should also be pointed out that our theorem implies Mahler's result of [Mah, page 365].

Given couples of algebraic numbers $\underline{u}_{1}, \ldots, \underline{u}_{m} \in \mathbb{G}_{m}^{2} \cap \mathcal{D}$ satisfying (1.4), our theorem gives an explicit and effective criterion to check whether the complex numbers $f_{\theta}\left(\underline{u}_{1}\right), \ldots, f_{\theta}\left(\underline{u}_{m}\right)$ are algebraically dependent or not; this property does not depend on $\underline{v}$ in (1.4), but only on $\underline{\zeta}_{1}, \ldots, \underline{\zeta}_{m}, \beta_{1}, \ldots, \beta_{m}$.

When the numbers $f_{\theta}\left(\underline{u}_{1}\right), \ldots, f_{\theta}\left(\underline{u}_{m}\right)$ are algebraically dependent, we are also able to determine the relations between them, as these can be deduced from the $\underline{\zeta}_{i}$ 's and the $\beta_{i}$ 's only.

We now give some explicit examples of relations and of applications of our theorem.

Example 1.3. Let us take any real number $w>0$ and two complex numbers $u, v$ such that $|u|<1$ and $0<|u||v|^{w}<1$. If we take $m=5$, then the following homogeneous linear relation can be easily verified:

$$
4 f_{w}\left(u^{2}, v^{2}\right)-f_{w}(u, v)-f_{w}(-u, v)-f_{w}(u,-v)-f_{w}(-u,-v)=0 .
$$

This relation is in some sense the simplest possible: it turns out that it is the shortest that holds, regardless of the algebraicity of $w, u, v$.

When $w$ is quadratic and irrational, we claim that the $m$-tuple

$$
\left(T .(u, v), T .(-u, v), T .(u,-v), T .(-u,-v), T .\left(u^{2}, v^{2}\right)\right)
$$

satisfies the rank one hypothesis; we prove it with $0<w<1$. Let us write $\underline{\zeta}_{2}=(-1,1), \underline{\zeta}_{3}=(1,-1)$ and $\underline{\zeta}_{4}=(-1,-1)$. Since $T \in \operatorname{SL}_{2}(\mathbb{Z})$, this matrix induces a permutation of the set $\left\{\underline{\zeta}_{2}, \underline{\zeta}_{3}, \underline{\zeta}_{4}\right\}$, that is: $T \cdot \underline{\zeta}_{i}=\underline{\zeta}_{\sigma(i)}(i=2,3,4)$ for 
a permutation $\sigma$ of the set $\{2,3,4\}$. Clearly, $2 \in S(M)$, and we find $\mathcal{B}(2)=\left(\begin{array}{ll}2 & 0 \\ 0 & 2\end{array}\right)$ in (1.3).

If we write $\underline{u}=(u, v), \underline{u}_{1}=\underline{v}=T \cdot \underline{u}, \underline{u}_{i}=T .\left(\underline{\zeta}_{i} \underline{u}\right),(i=2,3,4)$, and $\underline{u}_{5}=T \cdot\left(\underline{u}^{2}\right)$, we have

$$
\underline{u}_{1}=\underline{v}, \quad \underline{u}_{2}=\underline{\zeta}_{\sigma(2)} \underline{v}, \quad \underline{u}_{3}=\underline{\zeta}_{\sigma(3)} \underline{v}, \quad \underline{u}_{4}=\underline{\zeta}_{\sigma(4)} \underline{v}, \quad \underline{u}_{5}=\underline{v}^{2},
$$

hence proving the claim (we notice the distributive property: $T \cdot(\underline{a} \cdot \underline{b})=(T \cdot \underline{a})$. (T. $\underline{b})$ ); in particular, $\left(\underline{u}_{1}, \underline{u}_{2}, \underline{u}_{3}, \underline{u}_{4}\right)$ satisfies the rank one hypothesis.

By writing $\left(U_{1}, V_{1}\right)=(U, V),\left(U_{i}, V_{i}\right)=\underline{\zeta}_{\sigma(i)}(U, V)(i=2,3,4)$, it is easy to see that the formal double Laurent series

$$
\sum_{(l, h) \in \mathbb{Z}^{2} \backslash\{(0,0)\}} U_{i}^{l} V_{i}^{h}, \quad i=1, \ldots, 4
$$

are $\mathbb{Q}$-linearly independent so that, after the theorem, the four complex numbers $f_{w}(u, v), f_{w}(-u, v), f_{w}(u,-v), f_{w}(-u,-v)$ appearing in (1.8) are algebraically independent over $\mathbb{Q}$, and (1.8) is essentially the only algebraic relation between the five complex numbers appearing there.

Example 1.4. When $w=\theta$ satisfies (1.2), there also exist shorter relations (compare with [Mah, pages 363-366] and with [Ni, pages 42-45], especially the second equation on page 45; see also Section 3.5.3 of this article). Indeed, following [Ni, pages 42-45], if we write

$$
\mathcal{B}=\left(\begin{array}{rr}
b_{2 r} & 1 \\
1 & 0
\end{array}\right) \cdots\left(\begin{array}{rr}
b_{1} & 1 \\
1 & 0
\end{array}\right)=\left(\begin{array}{ll}
a & b \\
c & d
\end{array}\right)
$$

then

$$
\theta=\frac{d \theta+b}{c \theta+a}
$$

and

$$
f_{\theta}(\underline{u})-f_{\theta}(\mathcal{B} . \underline{u})=R(u, v)
$$

with $R \in \mathbb{Q}(u, v)$ explicitly described in [Ni]. This is a functional equation of $f_{\theta}$.

From (1.10) we get $\left(d+b \theta^{-1}\right) \theta^{-1}=c+a \theta^{-1}$, which implies that $\eta:=$ $d+b \theta^{-1} \in S(M)$ and $\mathcal{B}=\mathcal{B}(\eta)$. Now, since $\mathcal{B}(\eta) \in \mathrm{SL}_{2}(\mathbb{Z})$ by (1.9), we also see that $\eta^{-1} \in S(M)$ and $\eta$ is a unit in $S(M)$; with our notations:

$$
f_{\theta}(\underline{u})-f_{\theta}\left(\underline{u}^{\eta}\right)=R(u, v) .
$$

If we choose the smallest possible $r>0$ such that (1.6) holds, then it turns out that $\eta$ is the minimal irrational unit of $S(M)$ such that $\eta>1>\eta^{\prime}>0$ (that is, a unit of $S(M)$ such that for any other unit $\tilde{\eta} \in S(M)$ with $\tilde{\eta}>1>\tilde{\eta}^{\prime}>0$, 
there exists $s \in \mathbb{N}=\{0,1,2, \ldots\}$ such that $\left.\tilde{\eta}=\eta^{s}\right)$. If $u, v$ are algebraic, then we get linear relations, this time with $m=2$, because $R(u, v) \in \overline{\mathbb{Q}}$ (not necessarily homogeneous as $R(u, v)$ can be non-zero).

The couple $\left(\underline{u}, \underline{u}^{\eta}\right)$ satisfies the rank one hypothesis. If we write $\left(U_{1}, V_{1}\right)=$ $\left(U^{a} V^{b}, U^{c} V^{d}\right)$, then we check the identity in $\mathbb{C}\{\underline{U}\}=\mathbb{C}\{U, V\}$ :

$$
\sum_{(l, h) \in \mathbb{Z}^{2} \backslash\{(0,0)\}} U_{1}^{l} V_{1}^{h}=\sum_{(l, h) \in \mathbb{Z}^{2} \backslash\{(0,0)\}} U^{l} V^{h},
$$

because $\mathcal{B}(\eta) \in \mathrm{SL}_{2}(\mathbb{Z})$ and $\left(\mathbb{Z}^{2} \backslash\{0\}\right) \cdot \mathcal{B}(\eta)=\left(\mathbb{Z}^{2} \backslash\{0\}\right)$. This means that our theorem predicts the algebraic dependence of $f_{\theta}(\underline{u})$ and $f_{\theta}\left(\underline{u}^{\eta}\right)$, as it should be. We also notice that with the notations of Section 3.5.2, $R=R_{\eta}$.

If $\eta>1$ but $-1<\eta^{\prime}<0$, our theorem says that, again, $f_{\theta}(\underline{u})$ and $f_{\theta}\left(\underline{u}^{\eta}\right)$ are algebraically dependent, but this does not follow from (1.11). In Section 3.5.3 we describe the relations between these numbers (see (3.7) and (3.8)).

Example 1.5. We begin by pointing out that the Example 1.4 holds for irrational quadratic numbers $\theta$ satisfying (1.2), regardless of $K=\mathbb{Q}(\theta)$; we now give an example of a relation which holds for only one choice of $K$, and for $\theta \in K$ satisfying (1.2). Let us consider $K=\mathbb{Q}(\sqrt{2})$ and $\theta=\sqrt{2}-1$; clearly, it satisfies (1.2). For a couple of non-zero algebraic numbers $u, v$ such that $|u|<1$ and $0<|u||v|^{\theta}<1$, we have seen (Example 1.3) that $f_{\theta}(u, v), f_{\theta}(-u,-v)$ are algebraically independent. But with this choice of $\theta$, we also have

$$
f_{\theta}(u, v)+f_{\theta}(-u,-v)=2 f_{\theta}\left(u v, u v^{-1}\right) .
$$

We will prove this relation in Section 3.5.2: here we notice that $\sqrt{2}\left(\mathbb{Z}+\theta^{-1} \mathbb{Z}\right) \subset$ $\mathbb{Z}+\theta^{-1} \mathbb{Z}$ (thus $\sqrt{2} \in S(M)$ ), and $\left(u v, u v^{-1}\right)=(u, v)^{\sqrt{2}}$, so that the triple

$$
\left((u, v),(-u,-v),\left(u v, u v^{-1}\right)\right)=\left(\underline{u},-\underline{u}, \underline{u}^{\sqrt{2}}\right)
$$

satisfies the rank one hypothesis.

If we set $\underline{U}_{1}=\underline{U}, \underline{U}_{2}=-\underline{U}$ and $\underline{U}_{3}=\underline{U}^{\sqrt{2}}$, then

$$
\begin{aligned}
\sum_{(l, h) \in \mathbb{Z}^{2} \backslash\{(0,0)\}} U_{1}^{l} V_{1}^{h}+\sum_{(l, h) \in \mathbb{Z}^{2} \backslash\{(0,0)\}} U_{2}^{l} V_{2}^{h} & =2 \sum_{\substack{(l, h) \in \mathbb{Z}^{2} \backslash\{(0,0)\} \\
l+h \in 2 \mathbb{Z}}} U^{l} V^{h} \\
& =2 \sum_{(l, h) \in \mathbb{Z}^{2} \backslash\{(0,0)\}} U_{3}^{l} V_{3}^{h},
\end{aligned}
$$

so that, as expected, our theorem also predicts that $f_{\theta}(u, v), f_{\theta}(-u,-v)$, $f_{\theta}\left(u v, u v^{-1}\right)$ are algebraically dependent. The algebraic independence of these three numbers when $\theta$ is quadratic irrational not in $\mathbb{Q}(\sqrt{2})$ does not follow from the present work, but can be deduced from our results in [Pel2]. 
The following corollaries are consequences of our theorem; they will be proved in Section 7.

Corollary 1.6. Let $w>0$ be any quadratic irrational. Let $p$ be a positive integer, let $\zeta$ be a primitive $p$-th root of unity, let $(u, v)$ be an algebraic point of $\mathbb{G}_{\mathrm{m}}^{2}$ such that $|u|<1$ and $0<|u||v|^{w}<1$. Then, the numbers

$$
f_{w}\left(\zeta^{i} u, \zeta^{j} v\right), \quad 0 \leq i, j \leq p-1
$$

are algebraically independent over $\mathbb{Q}$.

Moreover, the $p^{2}+1$ numbers $f_{w}\left(\zeta^{i} u, \zeta^{j} v\right), 0 \leq i, j \leq p-1$ and $f_{w}\left(u^{p}, v^{p}\right)$ are algebraically dependent, and

$$
p^{2} f_{w}\left(u^{p}, v^{p}\right)=\sum_{0 \leq i, j \leq p-1} f_{w}\left(\zeta^{i} u, \zeta^{j} v\right)
$$

is the only non-trivial algebraic relation between them, up to a multiplication by a non-zero algebraic number.

The previous corollary generalises the Example 1.3.

Corollary 1.7. Let $\theta$ be a quadratic irrational satisfying (1.2). Let $\underline{v} \in \mathbb{G}_{m}^{2}$ be an algebraic point, let $\beta_{1}, \ldots, \beta_{m} \in S(M) \backslash\{0\}$ be such that if $i \neq j$, then $\beta_{i} / \beta_{j}$ is not a unit of $S(M)$, let us suppose that for all $i=1, \ldots, m, \underline{u}_{i}=\left(u_{i}, v_{i}\right):=\underline{v}^{\beta_{i}}$ satisfies $\left|u_{i}\right|<1$ and $0<\left|u_{i}\right|\left|v_{i}\right|^{\theta}<1$. Then the numbers $f_{\theta}\left(\underline{u}_{1}\right), \ldots, f_{\theta}\left(\underline{u}_{m}\right)$ are algebraically independent.

In particular, if $u, v$ are non-zero algebraic numbers such that $|u|<1$ and $|u||v|^{\theta}<1$, and if $\underline{u}=(u, v)$, the numbers

$$
f_{\theta}(\underline{u}), f_{\theta}\left(\underline{u}^{2}\right), \ldots, f_{\theta}\left(\underline{u}^{m}\right), \ldots
$$

are algebraically independent.

\subsection{Structure and methods of proof}

We describe the methods employed in this text. To prove our theorem, the main difficulty to overcome is the fact that we must work with non-entire analytic functions of two complex variables.

Mahler's method, when it applies, is an excellent technique for investigating arithmetic properties of functions of several variables. This is the way we approach our problem. Thus, the functional equation (1.11) will have a privileged role, and this explains why we need $w=\theta$ to be a quadratic irrational satisfying (1.2).

In order to describe our main ideas we begin by analysing a classical example. Let us consider algebraic numbers $u_{1}, \ldots, u_{m}$ such that $0<\left|u_{i}\right|<1$, and the 
Fredholm series $g(x)=\sum_{k=0}^{\infty} x^{2^{k}}$, satisfying the functional equation $g\left(x^{2}\right)=$ $g(x)-x$. In [Lo-Po3], the transcendence degree of the field $\mathbb{Q}\left(g\left(u_{1}\right), \ldots, g\left(u_{m}\right)\right)$ is explicitly computed.

The structure of the proof of this result is as follows. Let $\Gamma \subset \mathbb{G}_{\mathrm{m}}$ be the multiplicative group generated by the $u_{i}$ 's. Since they are not roots of unity, the rank $n$ of $\Gamma$ satisfies $1 \leq n \leq m$. It is possible to find multiplicatively independent elements $a_{1}, \ldots, a_{n} \in \Gamma$ such that $0<\left|a_{i}\right|<1$ for all $i$, and such that the following relations hold:

$$
u_{i}=\zeta_{i} a_{1}^{b_{i, 1}} \cdots a_{n}^{b_{i, n}}, \quad i=1, \ldots, m
$$

where $\zeta_{i}$ are roots of unity and $b_{i, j} \in \mathbb{N}$ for all $i, j$. Then, we consider the functions (analytic in $\underline{0}$ because of the positivity of the $b_{i, j}$ 's):

$$
\Psi_{i}\left(x_{1}, \ldots, x_{n}\right)=g\left(\zeta_{i} x_{1}^{b_{i, 1}} \ldots x_{n}^{b_{i, n}}\right), \quad i=1, \ldots, m
$$

Thanks to an algebraic independence criterion of Loxton and van der Poorten, we only need to classify algebraic dependence relations of the functions $\Psi_{i}$, and even better, linear dependence relations.

It also turns out that all the algebraic relations between numbers such as the $g(u)$ 's only arise in the "rank one case" $n=1$ (that is, when $u_{i}=\zeta_{i} a^{b_{i}}$ for some $a$ not a root of unity, and $\zeta_{i}$ a root of unity, for all $i$ ) or, equivalently, when the multiplicative group generated by the $u_{i}$ 's has rank one.

Let us now focus on our functions $f_{\theta}$ with $\theta$ satisfying (1.2). Let us choose algebraic points $\underline{u}_{1}, \ldots, \underline{u}_{m} \in \mathbb{G}_{\mathrm{m}}^{2}$ such that $f_{\theta}\left(\underline{u}_{1}\right), \ldots, f_{\theta}\left(\underline{u}_{m}\right)$ are well defined. We want to compute the transcendence degree of the field generated by $f_{\theta}\left(\underline{u}_{1}\right), \ldots, f_{\theta}\left(\underline{u}_{m}\right)$. It is natural to consider the multiplicative $S(M)$-module $\Gamma \subset$ $\mathbb{G}_{\mathrm{m}}^{2}$ generated by all the products $\underline{u}_{1}^{\beta_{1}} \cdots \underline{u}_{m}^{\beta_{m}}$ with $\beta_{i} \in S(M)$. We can define a notion of $S(M)$-rank for this module: let us denote it by $n$ again. We will check that the $\underline{u}_{i}$ 's are not couples of roots of unity, thus $1 \leq n \leq m$.

In analogy with the classical case of [Lo-Po3] sketched above, we must find elements $\underline{a}_{1}, \ldots, \underline{a}_{n} \in \Gamma, S(M)$-multiplicatively independent, close enough to the origin, with the property that relations as follows hold:

$$
\underline{u}_{i}=\underline{\zeta}_{i} \underline{a}_{1}^{\beta_{i, 1}} \cdots \underline{a}_{n}^{\beta_{i, n}}, \quad i=1, \ldots, m
$$

where $\underline{\zeta}_{i}$ are couples of roots of unity, the $\beta_{i, j}$ 's are elements of $S(M)$, and the product is the usual one in the algebraic group $\mathbb{G}_{m}^{2}$. This leads us to consider the following functions:

$$
\Psi_{i}\left(\underline{v}_{1}, \ldots, \underline{v}_{n}\right)=f_{w}\left(\underline{\zeta}_{i} \underline{v}_{1}^{\beta_{i, 1}} \cdots \underline{v}_{n}^{\beta_{i, n}}\right), \quad i=1, \ldots, m .
$$

In order to apply the criterion of Loxton and van der Poorten, we need the functions $\Psi_{i}$ to be analytic at $\underline{0}=(0,0)$. The validity of this property is ensured by the condition $\beta_{i, j} \geq\left|\beta_{i, j}^{\prime}\right|>0$ for all $i, j$. 
Since the time when the previously-mentioned result on the Fredholm series was proved, the criterion of Loxton and van der Poorten has been integrated in a more general result by Nishioka ([Ni, Theorem 3.3.2, page 88$]$ ); we refer to it from now on.

The key point of our work is precisely the following. While for Fredholm's series $g$, Loxton and van der Poorten studied the series

$$
g\left(\zeta_{i} v^{b_{i, 1}} \cdots v^{b_{i, n}}\right)
$$

with $\zeta_{i}$ roots of unity and with positive $b_{i, j}$, for Hecke-Mahler's series we study the series:

$$
f_{w}\left(\underline{\zeta}_{i} \underline{v}^{\beta_{i, 1}} \cdots \underline{v}^{\beta_{i, n}}\right)
$$

with couples of roots of unity $\underline{\zeta}_{i}$ and with "positive" $\beta_{i, j}$.

In spite of this analogy, our problem is only superficially similar to the corresponding problem on Fredholm's series; here is the main difference. By (1.3) the action of $S(M)$ depends on the choice of a basis of $M$ that we made. If we change the basis, we might change the validity of the rank one hypothesis and the linear independence properties of the formal double Laurent series involved (except admittedly, with the points involved in relation (1.8) or in Corollary 1.6).

This problem does not arise with Fredholm's series, where $\mathbb{Z}$ plays the role of $S(M)$, and where we have essentially one basis.

In this text, we only study the rank one case $n=1$ while the general case is studied in [Pel2]. There are three reasons for writing two texts instead of one.

We will prove in [Pel2] that all the relations among complex numbers (1.1) (with $w=\theta$ satisfying (1.2)) are generated by relations which hold in the rank one case (for example, relations (1.8), (1.11) and (1.12)), so that the rank one case needs special care and has to be analysed separately, before considering the general case.

Moreover, the construction of the functions $\Psi_{i}$ and the choice of the $\underline{a}_{i}$ 's with the properties above in the general case is quite complicated, so it is natural to consider it in a second text.

Finally, as for almost every transcendence or algebraic independence proof, we need a vanishing estimate. In the rank one case, Mahler's vanishing theorem (in [Mah]) is enough, but in the general case we will need Masser's vanishing theorem (cf. [Mas1]), and more work is needed to apply it to our context.

Here is the outline of the rest of this article. In Section 2 we give an account of the main tools (exponential functions, formal double Laurent series, rank one hypothesis...).

Several functions will appear in this text together with $f_{w}$. In Section 3 we present them with their main properties. In Section 4 we begin the study of linear independence of the functions introduced thus far. At the end of this section, we give a proof of the easiest part of the main theorem (algebraic independence implies linear independence of formal double Laurent series).

In Section 5 we give the most important tools in the proof of the more difficult implication in our theorem: the algebraic independence criterion given by $[\mathrm{Ni}$, Theorem 3.3.2, page 88] reduces the problem of checking algebraic independence of 
numbers to an algebraic independence problem of functions. Since these functions satisfy very simple functional equations, we are only concerned with a problem of linear independence of functions.

We first state and prove a simple topological argument which allows us to recognise whether a given series arises from an irrational function (Lemma 5.1). Then we consider certain functions $\phi_{i}$, and we use this topological argument to give equivalent conditions for their $\mathbb{C}$-linear independence (Proposition 5.7). In this part we use many properties related to the functional equation (1.11); in particular, we make use of several properties of "good fundamental domains" for the action of units. A technical difficulty appears; indeed we will also need to work with a "twin series" of $f_{\theta}$ (defined in Section 3.1). We do not know if we can avoid using this series.

In Section 6 (the conclusion) we state the above-mentioned criterion of algebraic independence; we then check all the hypotheses to make sure it applies to the functions $\Psi_{i}=\phi_{i}$.

\section{Basic tools about actions of orders of $K$ on $\mathbb{G}_{m}^{2}$}

We recall that $K \subset \mathbb{R}$ is a quadratic number field which is fixed once and for all, and that $\alpha \mapsto \alpha^{\prime}$ denotes its non-trivial automorphism. We also denote by $\overline{\mathbb{Q}}$ the algebraic closure of $\mathbb{Q}$ in $\mathbb{C}$ (thus $K \subset \overline{\mathbb{Q}}$ ).

All the objects that we are going to introduce here (stabilisers, exponential functions, etc.) depend on the choice of a complete module $M=B_{0} \mathbb{Z}+B_{1} \mathbb{Z} \subset K$ with a basis $\left(B_{0}, B_{1}\right)$ (a complete module is by definition, a free $\mathbb{Z}$-module of rank 2 contained in $K$; for the background about complete modules see [BS], chapter 2 ). We think that this level of generality will be useful for further references, but to prove our theorem we will only need to work with $\left(B_{0}, B_{1}\right)=\left(\theta^{-1}, 1\right)$ with $\theta \in K$ satisfying (1.2) so that $M=B_{0} \mathbb{Z}+B_{1} \mathbb{Z}=\theta^{-1} \mathbb{Z}+\mathbb{Z}$.

We denote by $\mathbb{G}_{\mathrm{m}}$ the complex multiplicative group $\mathbb{C}^{\times}:=\mathbb{C} \backslash\{0\}$. Let us also write:

$$
\mathbb{T}=\mathbb{G}_{\mathrm{m}}^{2},
$$

let us denote by $\underline{1}$ its identity element $(1,1)$.

All throughout this text the elements of $\mathbb{C}^{n}, \mathbb{R}^{n}, \ldots$ are considered as row matrices, unless otherwise specified.

\subsection{Exponential functions}

Let $\underline{z}=\left(z, z^{\prime}\right)$ be a couple of complex numbers. When both imaginary parts $\Im(z)$, $\Im\left(z^{\prime}\right)$ of $z, z^{\prime}$ are non-zero, we will often write:

$$
\mathbf{t}(\underline{z})=z+z^{\prime} .
$$

By abuse of notation, we will denote by $\mathbf{t}$ also the usual trace map $\mathbf{t}: K \rightarrow \mathbb{Q}$ of $K$ over $\mathbb{Q}$. If $\underline{z}=\left(z, z^{\prime}\right) \in \mathbb{C}^{2}$ and $v \in K$, we will write $v \underline{z}:=\left(v z, v^{\prime} z^{\prime}\right)$ 
and $v+\underline{z}=\left(v+z, v^{\prime}+z^{\prime}\right)$, so that, by our conventions: $\mathbf{t}(v \underline{z})=v z+v^{\prime} z^{\prime}$ and $\mathbf{t}(v+\underline{z})=\mathbf{t}(v)+\mathbf{t}(\underline{z})$.

Let $M$ be a complete module of $K$. We denote by $M^{*}$ the dual of $M$ for the trace t $: K \rightarrow \mathbb{Q}$, that is, the complete module of $K$ whose elements $v$ satisfy $\mathbf{t}(\mu \nu) \in \mathbb{Z}$ for all $\mu \in M$. If $M \subseteq N$ are complete modules, then $N^{*} \subseteq M^{*}$; If $N=v M$ for some $v \in K^{\times}:=K \backslash\{0\}$, then $N^{*}=v^{-1} M^{*}$.

Let $\Sigma: K \rightarrow \mathbb{R}^{2}$ be the embedding $\Sigma(v)=\left(v, v^{\prime}\right) \in \mathbb{R}^{2}$, let us fix a $\mathbb{Z}$-basis $\left(B_{0}, B_{1}\right)$ of $M$. We denote by $\left(B_{0}^{*}, B_{1}^{*}\right)$ the dual basis of $\left(B_{0}, B_{1}\right)$ for the trace and we write:

$$
\mathfrak{B}=\left(\begin{array}{ll}
B_{0}^{*} & B_{0}^{* \prime} \\
B_{1}^{*} & B_{1}^{* \prime}
\end{array}\right)=\left(\begin{array}{ll}
B_{0} & B_{1} \\
B_{0}^{\prime} & B_{1}^{\prime}
\end{array}\right)^{-1} .
$$

Together with the choice of the basis $\left(B_{0}, B_{1}\right)$ we have the exponential function with periods in $\Sigma(M)$ :

$$
\Phi: \mathbb{C}^{2} \rightarrow \mathbb{T}
$$

defined by

$$
\Phi\left(z, z^{\prime}\right)={ }^{\mathrm{t}} e\left(\mathfrak{B} \cdot{ }^{\mathrm{t}}\left(z, z^{\prime}\right)\right),
$$

where $^{\mathrm{t}}$. means "transpose", $e(\tau)=e(2 \pi \mathrm{i} \tau)(\mathrm{i}:=\sqrt{-1})$, and $e(a, b)=(e(a), e(b))$. In a more explicit way, $\Phi\left(z, z^{\prime}\right)=(u, v)$, where:

$$
u=e\left(B_{0}^{*} z+B_{0}^{* \prime} z^{\prime}\right), \quad v=e\left(B_{1}^{*} z+B_{1}^{* \prime} z^{\prime}\right) .
$$

This function $\Phi$ (which depends on the basis $\left(B_{0}, B_{1}\right)$ ) factors through $\mathbb{C}^{2} / \Sigma(M)$ because for complex numbers $z, z^{\prime}, \zeta, \zeta^{\prime}$ we have $\mathfrak{B} \cdot{ }^{\mathrm{t}}\left(z, z^{\prime}\right)-\mathfrak{B} \cdot{ }^{\mathrm{t}}\left(\zeta, \zeta^{\prime}\right) \in \mathbb{Z}^{2}$ if and only if $\left(z-\zeta, z^{\prime}-\zeta^{\prime}\right) \in \Sigma(M)$, and this happens if and only if $\Phi\left(z, z^{\prime}\right)=\Phi\left(\zeta, \zeta^{\prime}\right)$. Of course, if $\left(z, z^{\prime}\right),\left(\tilde{z}, \tilde{z}^{\prime}\right) \in \mathbb{C}^{2}$, then we have (product of the algebraic group $\mathbb{T}$ ):

$$
\Phi\left(z, z^{\prime}\right) \Phi\left(\tilde{z}, \tilde{z}^{\prime}\right)=\Phi\left(z+\tilde{z}, z^{\prime}+\tilde{z}^{\prime}\right) .
$$

\subsection{Actions of orders of $K$ on $\mathbb{T}$}

Let us consider the stabiliser $S(M)$ of $M$, i. e. the order of $K$ whose elements are the $\beta$ 's such that $\beta M \subset M$. We notice that if $v \in K^{\times}$, then $S(v M)=S(M)$. Moreover, $S\left(M^{*}\right)=S(M){ }^{2}$ from now on, we will write $S=S(M)$.

Let $\beta$ be an element of $S$, let $\mathcal{B}(\beta)$ be the matrix of the natural multiplicative $S$-action on $M$, expressed with the basis $\left(B_{0}, B_{1}\right)$, that is, the matrix $\left(\begin{array}{ll}a & b \\ c & d\end{array}\right)$ with $a, b, c, d$ as in (1.3). We have

$$
\mathcal{B}(\beta)=\mathfrak{B} \cdot\left(\begin{array}{ll}
\beta & 0 \\
0 & \beta^{\prime}
\end{array}\right) \cdot \mathfrak{B}^{-1} .
$$

2 The stabiliser $S(M)$ can be computed explicitly. For instance, it is well known that $S(M)$ is a complete module $\mathbb{Z}+\kappa \mathbb{Z}$ with $\kappa$ an irrational integer of $K$. Moreover, by [BS, Exercise 17, Chapter 2], $S(M)=\left(M M^{*}\right)^{*}$. 
Clearly, $\mathcal{B}(\beta)$ has determinant $\mathbf{n}(\beta)$ (where $\mathbf{n}(\beta)$ is the norm $\beta \beta^{\prime}$ of $\beta$ over $\mathbb{Q}$ ) and has rational integer entries.

As in the introduction, the multiplicative group $\mathbb{T}$ is endowed with an action of $S$ defined as follows. Let $\underline{u}=(u, v) \in \mathbb{T}$ and let $\beta$ be an element of $S$; then we define:

$$
\underline{u}^{\beta}:=\mathcal{B}(\beta) . \underline{u} .
$$

If $\underline{u}=\Phi\left(z, z^{\prime}\right)$ then, by (2.4), $\underline{u}^{\beta}=\Phi\left(\beta z, \beta^{\prime} z^{\prime}\right)$. This action depends on $M$ and on the basis $\left(B_{0}, B_{1}\right)$. Nevertheless, if $\beta \in \mathbb{Z}$, then the action

$$
\underline{u}^{\beta}=\left(u^{\beta}, v^{\beta}\right)
$$

does not even depend on $K$.

We recall that a point $\underline{u} \in \mathbb{T}$ is a torsion point if $\underline{u}=\left(\zeta_{1}, \zeta_{2}\right)$ with $\zeta_{1}, \zeta_{2}$ roots of unity. A point which is not a torsion point is said to be a point of infinite order.

Lemma 2.1. A point $\underline{u} \in \mathbb{T}$ is a torsion point if and only if

$$
\underline{u} \in \Phi(\Sigma(K)),
$$

that is, if there exists $\alpha \in K$ such that $\underline{u}=\Phi\left(\alpha, \alpha^{\prime}\right)$. If $\Phi\left(\alpha, \alpha^{\prime}\right)=\Phi\left(\tilde{\alpha}, \tilde{\alpha}^{\prime}\right)$ for some $\alpha, \tilde{\alpha} \in K$, then $\alpha-\tilde{\alpha} \in M$.

Proof. Let us notice that given a complete module $N$, if $\alpha \in K^{\times}$then $\ell \alpha \in N$ for some $\ell \in \mathbb{N} \backslash\{0\}$ (to check this, it is enough to choose a basis $N=\tilde{B}_{0} \mathbb{Z}+\tilde{B}_{1} \mathbb{Z}$ and write $\alpha=r_{1} \tilde{B}_{0}+r_{2} \tilde{B}_{1}$ with $r_{1}, r_{2} \in \mathbb{Q}$ ).

Thus, by (2.5),

$$
\Phi\left(\alpha, \alpha^{\prime}\right)^{\ell}=\Phi\left(\ell \alpha, \ell \alpha^{\prime}\right)=\underline{1},
$$

which proves that $\Phi\left(\alpha, \alpha^{\prime}\right)$ is a torsion point. Let now $(\zeta, \varsigma)=e(a, b)$ be a torsion point of $\mathbb{T}(a, b \in \mathbb{Q})$; we have $\Phi\left(\alpha, \alpha^{\prime}\right)=(\zeta, \varsigma)$ with $\alpha=B_{0} a+B_{1} b$ by (2.3).

The second assertion is clear because $1=\Phi\left(\alpha, \alpha^{\prime}\right) \Phi\left(\tilde{\alpha}, \tilde{\alpha}^{\prime}\right)^{-1}=\Phi(\alpha-$ $\left.\tilde{\alpha}, \alpha^{\prime}-\tilde{\alpha}^{\prime}\right)$ so that $\left(\alpha-\tilde{\alpha}, \alpha^{\prime}-\tilde{\alpha}^{\prime}\right)$ lies inside the set of periods of $\Phi$, which is $\Sigma(M)$.

The subgroup of $\mathbb{T}$ whose elements are torsion points is denoted by $\mathbb{T}_{\text {tors }}$. A point $\underline{u} \in \mathbb{T}$ is torsion if and only if there exists $\beta \in S \backslash\{0\}$ such that $\underline{u}^{\beta}=\underline{1}$. The set of elements $\beta \in S$ such that $\underline{u}^{\beta}=1$, which is a ring containing $\bar{p} \mathbb{Z}$ for some integer $p>0$, depends on $\Phi$, but the property of being a torsion point does not.

\subsection{Rank one hypothesis}

We fix an exponential function $\Phi$ as in (2.2). Let us consider an $m$-tuple $\mathcal{M}=$ $\left(\underline{u}_{1}, \ldots, \underline{u}_{m}\right) \in \mathbb{T}^{m}$ of points of infinite order.

We say that $\mathcal{M}$ satisfies the rank one hypothesis with respect to $\Phi$ if there is a point of infinite order $\underline{v} \in \mathbb{T}, \alpha_{1}, \ldots, \alpha_{m} \in K$ and $\beta_{1}, \ldots, \beta_{m} \in S \backslash\{0\}$, such that for all $i=1, \ldots, m$ :

$$
\underline{u}_{i}=\Phi\left(\alpha_{i}, \alpha_{i}^{\prime}\right) \underline{v}^{\beta_{i}} .
$$


Remark 2.2. The $m$-tuple $\mathcal{M}$ satisfies the rank one hypothesis with respect to $\Phi$ if and only if the $S$-module generated by $\underline{u}_{1}, \ldots, \underline{u}_{m}$ has rank one; in this case the point $\underline{v}$ in (2.7) is a generator, modulo torsion.

\subsection{A special choice for $\left(B_{0}, B_{1}\right)$}

Let us suppose right away that $w=\theta$ satisfies (1.2). Let $M=\mathbb{Z}+\theta^{-1} \mathbb{Z}$ be the complete module with the basis $\left(B_{0}, B_{1}\right)=\left(\theta^{-1}, 1\right)$. The matrix $\mathfrak{B}$ of $(2.1)$ becomes:

$$
\mathfrak{B}=\left(\begin{array}{ll}
B_{0}^{*} & B_{0}^{* \prime} \\
B_{1}^{*} & B_{1}^{* \prime}
\end{array}\right)=\delta^{-1}\left(\begin{array}{cc}
1 & -1 \\
-\theta^{\prime-1} & \theta^{-1}
\end{array}\right),
$$

where $\delta:=\theta^{-1}-\theta^{\prime-1}$ (notice that $\delta^{\prime}=-\delta$ ). The exponential function $\Phi$ becomes:

$$
\Phi\left(z, z^{\prime}\right)={ }^{\mathrm{t}} e\left(\mathfrak{B} \cdot{ }^{\mathrm{t}}\left(z, z^{\prime}\right)\right)=e\left(\delta^{-1}\left(z-z^{\prime}\right), \delta^{-1}\left(-\theta^{\prime-1} z+\theta^{-1} z^{\prime}\right)\right)
$$

The number satisfying (1.2) will be fixed in the following; hence we write from now on

$$
f(\underline{u})=f_{\theta}(\underline{u}) .
$$

\section{Basic tools concerning series}

Here is the content of this section.

First of all, we introduce a "twin series" $f^{+}$of $f$ (see Section 3.1). After this, we will be mainly concerned with "formal series associated to sectors". Let $Z$ be a subgroup of $\mathbb{Z}^{2}$ of finite index. We are interested in the subset of $\mathbb{C}\{\underline{U}\}$ of series of the type

$$
f_{\mathcal{C}}^{Z}(\underline{U})=\sum_{(l, h) \in \mathcal{C} \cap Z} U^{l} V^{h},
$$

where $\mathcal{C}$ is a "sector" of $\mathbb{R}^{2} \backslash\{(0,0)\}$ (see Section 3.2); examples of these series are $f$ and $f^{+}$.

We will study under which conditions on $\mathcal{C}$ and $Z$, the series $f_{\mathcal{C}}^{Z}$ converges to a rational function of $\mathbb{Q}(\underline{u})$ (Section 3.3).

Each element $\beta \in S \backslash\{0\}$ determines maps on the subset of $\mathbb{C}\{\underline{U}\}$ of series of type $f_{\mathcal{C}}^{Z}$; for example:

$$
f_{\mathcal{C}}^{Z}(\underline{U}) \mapsto f_{\mathcal{C}}^{Z}\left(\underline{U}^{\beta}\right), \quad f_{\mathcal{C}}^{Z}(\underline{U}) \mapsto f_{\mathcal{C}}^{Z \cdot \mathcal{B}(\beta)}(\underline{U})
$$

To have a satisfactory picture of the effects of these maps, it is convenient to study a space of "formal Fourier series" that we introduce in Section 3.4.

We will apply our results to some special examples of series $f_{\mathcal{C}}^{Z}$ required to prove our theorem (in Section 3.5). 


\subsection{The twin series $f^{+}$}

Since $\theta$ satisfies (1.2), we have $\theta<2 / \mathbf{t}\left(\theta^{-1}\right)$. The twin series $f^{+}$of $f$ is the series:

$$
f^{+}(\underline{u})=\sum_{l=1}^{\infty} \sum_{h=[\theta l]+1}^{\left\lfloor\left(2 / \mathbf{t}\left(\theta^{-1}\right)\right) l\right\rfloor} u^{l} v^{h},
$$

where $\lfloor x\rfloor$ denotes the biggest integer $r$ such that $r<x$ (following our conventions, if for some $l$ the sum over $h$ has no terms, then its contribution to the series is zero). Since

$$
f^{+}(u, v)=f_{2 / \mathbf{t}\left(\theta^{-1}\right)}(u, v)-f(u, v)-\sum_{i=1}^{\infty}\left(u^{q} v^{p}\right)^{i}
$$

where $p, q$ are coprime positive integers such that $2 / \mathbf{t}\left(\theta^{-1}\right)=p / q$ and since $f(u, v), \sum_{i=1}^{\infty}\left(u^{q} v^{p}\right)^{i}$ are subseries of $f_{2 / \mathbf{t}\left(\theta^{-1}\right)}(u, v)$, it is easy to show that the series $f^{+}$converges on the domain:

$$
\mathcal{D}^{+}=\left\{(u, v) \in \mathbb{C}^{2} \text { such that }|u|<1 \text { and }|u|^{p}|v|^{q}<1\right\} .
$$

We recall that we have denoted by $\mathcal{D}$ the domain of convergence of $f$; the domain $\mathcal{D}$ contains $\mathcal{D}^{+}$.

We will see (in Section 3.3) that the series $f_{2 / \mathbf{t}\left(\theta^{-1}\right)}(u, v)$ converges to a rational function of $\mathbb{Q}(u, v)$. Thus, $f^{+}$differs from $-f$ by a rational function.

\subsection{Sectors}

A subset $\mathcal{C}$ of $\mathbb{R}^{2} \backslash\{(0,0)\}$ is a sector if there exists a non-empty connected subset $J \subseteq\left\{(x, y) \in \mathbb{R}^{2}\right.$ with $\left.x^{2}+y^{2}=1\right\}$ such that $\mathcal{C}=\left\{(\alpha x, \alpha y)\right.$ with $\alpha \in \mathbb{R}_{>0}$ and $(x, y) \in J\}$. Equivalently, we may ask $\mathcal{C}$ to be: stable under $(x, y) \mapsto(\alpha x, \alpha y)$ for $\alpha \in \mathbb{R}_{>0}$, not containing 0 , and connected.

Let $\mathcal{A}: \mathbb{R}^{2} \rightarrow \mathbb{R}^{2}$ be an $\mathbb{R}$-linear automorphism, let $\mathcal{C}$ be a sector; then $\mathcal{A}(\mathcal{C})$ is also a sector (it is connected, does not contain 0 , and is stable under multiplication by strictly positive numbers).

Let $I$ be a subset of $\mathbb{R}$, let us write:

$$
\mathcal{C}(I)=\left\{(x, y) \in \mathbb{R}_{>0} \times \mathbb{R} \text { such that } y=\alpha x \text { for } \alpha \in I\right\} .
$$

If $I$ is connected (an interval), then $\mathcal{C}(I)$ and $\mathcal{A}(\mathcal{C}(I))$ are sectors. In particular, the discussion above applies to the automorphism $\mathcal{A}$ defined by

$$
(x, y) \mapsto(x, y) \cdot \mathfrak{B}^{-1}=\left(x \theta^{-1}+y \theta^{-1}, x+y\right) .
$$

We adopt standard notations for the intervals with extremities $a, b$ (with $a \in \mathbb{R} \cup$ $\{-\infty\}$ and $b \in \mathbb{R} \cup\{+\infty\}$ such that $a \leq b)$ :

$$
[a, b],[a, b[,] a, b],] a, b[\text {. }
$$

For example, $[a, b[=\{c \in \mathbb{R}$ such that $a \leq c<b\}$, and if $b=+\infty,[a,+\infty[=$ $\{c \in \mathbb{R}$ such that $c \geq a\}$. 
Lemma 3.1. We have the following facts.

1. Let I be a subset of $\mathbb{R}$; then, $I \subseteq]-\infty,-\theta^{\prime} / \theta\left[\right.$ if and only if $\mathcal{C}(I) \cdot \mathfrak{B}^{-1} \subset$ $\mathbb{R}_{>0} \times \mathbb{R}$.

2. We have that $I \subseteq]-1,-\theta^{\prime} / \theta\left[\right.$ if and only if $\mathcal{C}(I) \cdot \mathfrak{B}^{-1} \subseteq \mathbb{R}_{>0} \times \mathbb{R}_{>0}$.

3. Let us consider the function $\rho:\left[-\infty,-\theta^{\prime} / \theta\right] \rightarrow\left[\theta^{\prime},+\infty\right]$ defined by

$$
\rho(z)=\frac{1+z}{\theta^{-1}+\theta^{\prime-1} z}
$$

on $]-\infty,-\theta^{\prime} / \theta\left[\right.$, and by $\rho(-\infty)=\theta^{\prime}, \rho\left(-\theta^{\prime} / \theta\right)=+\infty$. Then $\rho$ is a strictly increasing continuous function, and we have the following table of values:

\begin{tabular}{|l|ccccc|}
\hline$\alpha$ & $-\infty$ & -1 & 0 & 1 & $-\theta^{\prime} / \theta$ \\
\hline$\rho(\alpha)$ & $\theta^{\prime}$ & 0 & $\theta$ & $2 / \mathbf{t}\left(\theta^{-1}\right)$ & $+\infty$ \\
\hline
\end{tabular}

4. If $I \subseteq]-\infty,-\theta^{\prime} / \theta[$ is an interval $[a, b]$ (respectively $\left.] a, b\right],[a, b[$, ] $a, b[$ ) with $a, b \in\left[-\infty,-\theta^{\prime} / \theta\right]$ satisfying $a<b$, then $\mathcal{C}(I) \cdot \mathfrak{B}^{-1}=\mathcal{C}\left(I^{*}\right)$, where $I^{*}$ is the interval $[\rho(a), \rho(b)]$ (respectively $] \rho(a), \rho(b)],[\rho(a), \rho(b)[,] \rho(a), \rho(b)[)$.

\section{Proof.}

1. Let $y=\alpha x$ with $x>0$ and $\alpha \in I$. After (1.2), the first coordinate of $(x, y) \cdot \mathfrak{B}^{-1}$ is positive if and only if $x>-\left(\theta / \theta^{\prime}\right) y=-\left(\theta / \theta^{\prime}\right) \alpha x$, that is, $\alpha<-\theta^{\prime} / \theta$ because after (1.2) again, $-\theta / \theta^{\prime}>0$.

2. The second coordinate of $(x, y) \cdot \mathfrak{B}^{-1}$ is positive if and only if $x>-y=-\alpha x$. After the first property, both coordinates of $(x, y) \cdot \mathfrak{B}^{-1}$ are positive (for $x>0$ ) if and only if $-1<\alpha<-\theta^{\prime} / \theta$ for all $\alpha \in I$.

3. It follows from (1.2) that $1<-\theta^{\prime} / \theta$ and $\theta<2 / \mathbf{t}\left(\theta^{-1}\right)$. The properties of $\rho$ are obvious because

$$
\frac{d \rho}{d z}=\frac{\theta \theta^{\prime}\left(\theta^{\prime}-\theta\right)}{\left(\theta z+\theta^{\prime}\right)^{2}}
$$

is well defined and $>0$ after (1.2), if $z \neq-\theta^{\prime} / \theta$. Moreover, right and left limits at the extremities of the interval $]-\infty,-\theta^{\prime} / \theta$ [ agree with the given values. The table of values is easy to check.

4. This follows straightforwardly from the previous properties; the function $\rho$ has the property that if $\alpha=y / x$ then $\rho(\alpha)$ is the slope of the line through $(0,0)$ and the point $(x, y) \cdot \mathfrak{B}^{-1}$.

\subsection{Formal double Laurent series associated to sectors}

Let $\mathcal{C}$ be a sector, let $Z \subset \mathbb{Z}^{2}$ be a subgroup of finite index; we now study the formal series

$$
f_{\mathcal{C}}^{Z}(U, V)=\sum_{(l, h) \in Z \cap \mathcal{C}} U^{l} V^{h} \in \mathbb{C}\{\underline{U}\} .
$$


Examples. We have

$$
f=f_{\mathcal{C}(] 0, \theta[)}^{\mathbb{Z}^{2}}, \quad f^{+}=f_{\mathcal{C}(] \theta, 2 / \mathbf{t}\left(\theta^{-1}\right)[]}^{\mathbb{Z}^{2}}
$$

Clearly, $\mathbb{R}^{2} \backslash\{(0,0)\}$ is a sector, and

$$
f_{\mathbb{R}^{2} \backslash\{(0,0)\}}^{\mathbb{Z}^{2}}(\underline{U})=\sum_{(l, h) \in \mathbb{Z}^{2} \backslash\{(0,0)\}} U^{l} V^{h} .
$$

The series (1.5) of our theorem are nothing but the series $\left.f_{\mathbb{R}^{2} \backslash\{(0,0)\}}^{\mathbb{Z}^{2}} \underline{(\zeta} \underline{U}^{\beta}\right)$ with $\underline{\zeta} \in \mathbb{T}_{\text {tors }}$ and $\beta \in S \backslash\{0\}$; later, when we will use these series, we will employ the notation $\mathbb{F}=f_{\mathbb{R}^{2} \backslash\{(0,0)\}}^{\mathbb{Z}^{2}}$.

In the following lemma, we study some rationality properties of the series $f_{\mathcal{C}}^{Z}$. Let us denote by $\mathfrak{h}$ the open half-plane of $\mathbb{R}^{2} \backslash\{(0,0)\}$ having the line $h=\theta^{\prime} l$ as a boundary and containing the point $(1,1)$.

Let us consider a sector $\mathcal{C} \subset \mathfrak{h}$ such that its closure is contained in $\mathfrak{h} \cup\{(0,0)\}$. In particular, the boundary of $\mathcal{C}$ is the union of two half-lines $l_{1}, l_{2} \subset \mathfrak{h} \cup\{(0,0)\}$ (necessarily non-parallel) both containing $(0,0)$.

It can be proved that there exists a non-empty open subset $\mathcal{O} \subset \mathbb{C}^{2}$ on which $f_{\mathcal{C}}^{Z}$ converges (see the remark after the following lemma) but here, to simplify our exposition, we will assume this as an hypothesis: it will be enough for the applications in view.

Lemma 3.2. Let us suppose:

1. that $Z=\mathbb{Z}^{2} \cdot A$, where $A$ is a non-singular matrix with integer entries such that $\mathfrak{h} \cdot A=\mathfrak{h}$,

2. that the slopes of $l_{1}, l_{2}$ are rational,

3. that there exists a non-empty open subset $\mathcal{O} \subset \mathbb{C}^{2}$ on which $f_{\mathcal{C}}^{Z}$ converges.

Then, on $\mathcal{O}, f_{\mathcal{C}}^{Z}$ converges to a rational function of $\mathbb{Q}(u, v)$.

Proof. Let us first consider the case $A=$ identity matrix, so that $Z=\mathbb{Z}^{2}$. For $\left(p_{1}, q_{1}\right),\left(p_{2}, q_{2}\right)$ couples of coprime rational integers such that $\left(p_{1}, q_{1}\right) \in l_{1}$ and $\left(p_{2}, q_{2}\right) \in l_{2}$, we consider the closed parallelogram $\mathcal{P}$ of vertices $(0,0),\left(p_{2}, q_{2}\right)$, $\left(p_{1}+p_{2}, q_{1}+q_{2}\right)$ and $\left(p_{1}, q_{1}\right)$, the closed segments $\mathcal{S}_{1}, \mathcal{S}_{2}$ of extremities $(0,0)$, $\left(p_{1}, q_{1}\right)$ and $(0,0),\left(p_{2}, q_{2}\right)$, and the domain $\mathcal{R}:=\mathcal{P} \backslash\left(\mathcal{S}_{1} \cup \mathcal{S}_{2}\right)$.

Let us write $P(u, v)=\sum_{(l, h) \in \mathcal{R} \cap \mathbb{Z}^{2}} u^{l} v^{h}$; it is a Laurent polynomial because $\mathbb{Z}^{2}$ is discrete in $\mathbb{R}^{2}$ and $\mathcal{R}$ is bounded. On $\mathcal{O}$ we have

$$
f_{\mathcal{C}}^{\mathbb{Z}^{2}}(u, v)=\sum_{n_{1}=0}^{\infty} \sum_{n_{2}=0}^{\infty}\left(u^{p_{1}} v^{q_{1}}\right)^{n_{1}}\left(u^{p_{2}} v^{q_{2}}\right)^{n_{2}} P(u, v)
$$


so that on $\mathcal{O}$, the function

$$
\left(1-u^{p_{1}} v^{q_{1}}\right)\left(1-u^{p_{2}} v^{q_{2}}\right) f_{\mathcal{C}}^{Z}(u, v)
$$

is equal to $P(u, v)$, hence rational; this implies that $f_{\mathcal{C}}^{Z}$ converges to a rational function of $\mathbb{Q}(u, v)$ on $\mathcal{O}$.

If $Z \neq \mathbb{Z}^{2}$, we observe that there exists, thanks to the hypothesis on $A$, another sector $\tilde{\mathcal{C}} \subset \mathfrak{h}$ such that $\tilde{\mathcal{C}} \cdot A=\mathcal{C}$. The boundary of this sector is the union of the boundaries of two half-lines $\tilde{l}_{1}, \tilde{l}_{2} \subset \mathfrak{h}$ with rational slopes. The discussion above implies that over some non-empty open subset of $\mathbb{T}, f_{\tilde{\mathcal{C}}}^{\mathbb{Z}^{2}}$ converges to a rational function of $\mathbb{Q}(u, v)$. Since $f_{\mathcal{C}}^{Z}=f_{\tilde{\mathcal{C}} \cdot A}^{\mathbb{Z}^{2} \cdot A}(\underline{U})=f_{\tilde{\mathcal{C}}}^{\mathbb{Z}^{2}}(A \cdot \underline{U})$, the property follows for $f_{\mathcal{C}}^{Z}$.

Remark 3.3. It can be proved that if a sector $\mathcal{C}$ does not contain any half-plane, then there exists a non-empty open subset $\mathcal{O} \subset \mathbb{T}$ such that $f_{\mathcal{C}}^{Z}$ converges on $\mathcal{O}$; we do not need this property here.

\subsection{Formal Fourier series associated to sectors}

An element $v$ of $K$ belongs to $M^{*}$ if and only if

$$
v=h B_{1}^{*}+l B_{0}^{*}=\delta^{-1}\left(-h \theta^{\prime-1}+l\right)
$$

with $l, h \in \mathbb{Z}$ (unique). We can write (3.1) in the following equivalent way:

$$
\left(v, v^{\prime}\right)=(l, h) \cdot \mathfrak{B},
$$

from which we deduce that if $(U, V)=\Phi\left(Z, Z^{\prime}\right)$, then

$$
U^{l} V^{h}=e(\mathbf{t}(\nu \underline{Z})) .
$$

Let

$$
\Omega\left(Z, Z^{\prime}\right)=\sum_{\nu \in M^{*}} c_{\nu} e(\mathbf{t}(\nu \underline{Z})),
$$

be a formal Fourier series, let us write $M(\underline{U})^{v}=U^{l} V^{h}$ where $v, l, h$ are related to each other by (3.1). To $\Omega$ is associated a formal double Laurent series $\omega$ in the variables $U, V$, defined by:

$$
\omega(\underline{U})=\sum_{\nu \in M^{*}} c_{\nu} M(\underline{U})^{v} .
$$

If $\underline{u}=\Phi\left(z, z^{\prime}\right)$, then $\Omega$ converges at $\left(z, z^{\prime}\right)$ if and only $\omega$ converges at $\underline{u}$, and $\omega(\underline{u})=\Omega\left(z, z^{\prime}\right)$.

Let $\Omega$ be as in (3.4). The $K$-support $\Sigma_{K}(\Omega)$ of $\Omega$ is the subset of $\Sigma\left(M^{*}\right) \subset \mathbb{R}^{2}$ whose elements are the $\left(v, v^{\prime}\right)$ 's such that $c_{\nu} \neq 0$. Let $\omega$ be associated to $\Omega$ as 
above. The $K$-support $\Sigma_{K}(\omega)$ of $\omega$ is equal, by definition, to the $K$-support of $\Omega$. By definition again, the $K$-support of the zero series is the empty set $\varnothing$.

Let $\mathcal{F}$ be a sector, let $N \supseteq M$ be a complete module, let us consider the formal Fourier series

$$
F_{\mathcal{F}}^{N}\left(Z, Z^{\prime}\right)=\sum_{\left(\nu, v^{\prime}\right) \in \mathcal{F} \cap \Sigma\left(N^{*}\right)} e(\mathbf{t}(\nu \underline{Z})) .
$$

There is a one-to-one correspondence between series of the type $f_{\mathcal{C}}^{Z}$ with $\mathcal{C} \subset \mathfrak{h}$ and series of the type $F_{\mathcal{F}}^{N}$ with $\mathcal{F} \subset \mathfrak{H}:=\{(x, y)$ such that $x>0\}$, as the following lemma says.

Lemma 3.4. Let $\underline{U},\left(Z, Z^{\prime}\right)$ be such that $\Phi\left(Z, Z^{\prime}\right)=\underline{U}$, let us consider an element $\beta \in S \backslash\{0\}$ and let us write $N=\beta^{-1} M$. Let $\mathcal{F}$ be a sector. Then,

$$
F_{\mathcal{F}}^{N}\left(Z, Z^{\prime}\right)=f_{\mathcal{F} \cdot \mathfrak{B}^{-1}}^{\mathbb{Z}^{2} \cdot \mathcal{B}(\beta)}(\underline{U})
$$

Proof. We have, by using (3.2) and (2.4),

$$
\left(\beta v, \beta^{\prime} v^{\prime}\right)=(l, h) \cdot \mathfrak{B} \cdot\left(\begin{array}{cc}
\beta & 0 \\
0 & \beta^{\prime}
\end{array}\right)=(l, h) \cdot \mathcal{B}(\beta) \cdot \mathfrak{B} .
$$

Thus, $\Sigma\left(N^{*}\right)=\mathbb{Z}^{2} \cdot \mathcal{B}(\beta) \cdot \mathfrak{B}$ and we obtain (taking into account (3.3)):

$$
F_{\mathcal{F}}^{N}\left(Z, Z^{\prime}\right)=\sum_{\nu \in \mathcal{F} \cap \Sigma\left(N^{*}\right)} M(\underline{U})^{v}=\sum_{(l, h) \in \mathcal{F} \cdot \mathfrak{B}^{-1} \cap \mathbb{Z}^{2} \cdot \mathcal{B}(\beta)} U^{l} V^{h},
$$

which proves the lemma.

\subsection{Series associated to some special sectors}

We now introduce the series which are behind the construction of the numbers $\lambda$ in our theorem.

3.5.1. The series $f_{N}, f_{N}^{+}, \Theta_{N}$

Let $\beta>0$ be an element of $S \backslash\{0\}$, let us write $N=\beta^{-1} M$ and $Z=\mathbb{Z}^{2} \cdot \mathcal{B}(\beta)$. We consider the formal series

$$
\begin{aligned}
f_{N}(\underline{U}) & =f_{\mathcal{C}(] 0, \theta[)}^{Z}(\underline{U}), \\
f_{N}^{+}(\underline{U}) & =f_{\mathcal{C}(] \theta, 2 / \mathbf{t}\left(\theta^{-1}\right)[)}^{Z}(\underline{U}), \\
\Theta_{N}(\underline{U}) & =f_{\mathcal{C}(] 0,2 / \mathbf{t}\left(\theta^{-1}\right)[)}^{Z}(\underline{U}) .
\end{aligned}
$$


Proposition 3.5. With the notations above, we have the following properties.

1. If $N=M$, then $f_{M}=f$ and $f_{M}^{+}=f^{+}$.

2. We have the equality of formal series $\Theta_{N}=f_{N}+f_{N}^{+}$.

3. The series $f_{N}$ converges on $\mathcal{D}$ and the series $f_{N}^{+}, \Theta_{N}$ converge on $\mathcal{D}^{+}$. in particular, $f_{N}, f_{N}^{+}, \Theta_{N}$ simultaneously converge on $\mathcal{D}^{+}$.

4. On $\mathcal{D}^{+}$, the series $\Theta_{N}$ converges to a rational function of $\mathbb{Q}(\underline{u})$.

5. If $\underline{u}=\Phi\left(z, z^{\prime}\right)$, we have the following equalities of convergent series:

$$
\begin{array}{rlrl}
f_{N}(\underline{u}) & =F_{\mathcal{C}(]-1,0[)}^{N}\left(z, z^{\prime}\right), \quad \underline{u} \in \mathcal{D} \\
f_{N}^{+}(\underline{u}) & =F_{\mathcal{C}(] 0,1[)}^{N}\left(z, z^{\prime}\right), & \underline{u} & \in \mathcal{D}^{+} \\
\Theta_{N}(\underline{u}) & =F_{\mathcal{C}(]-1,1[)}^{N}\left(z, z^{\prime}\right) & \underline{u} & \in \mathcal{D}^{+} .
\end{array}
$$

6. If $\beta \in S \backslash\{0\}$ is such that $\beta \geq\left|\beta^{\prime}\right|>0$ and if $\zeta$ is a torsion point, the series $f_{N}\left(\underline{\zeta}^{\beta}\right), f_{N}^{+}\left(\underline{\zeta}^{\beta}\right)$ and $\Theta_{N}\left(\underline{\zeta}^{\beta}\right)$ converge for $\underline{u} \in \mathcal{D}^{+}$. More explicitly, we have the following table which describes domains where the considered series certainly converge:

\begin{tabular}{|c|c|c|c|}
\hline & $f_{N}\left(\underline{\zeta} \underline{\underline{u}} \underline{u}^{\beta}\right)$ & $f_{N}^{+}\left(\underline{\zeta} \underline{u}^{\beta}\right)$ & $\Theta_{N}\left(\underline{\zeta} \underline{u}^{\beta}\right)$ \\
\hline$\beta \geq \beta^{\prime}>0$ & $\mathcal{D}$ & $\mathcal{D}^{+}$ & $\mathcal{D}^{+}$ \\
\hline$\beta \geq-\beta^{\prime}>0$ & $\mathcal{D}^{+}$ & $\mathcal{D}$ & $\mathcal{D}^{+}$ \\
\hline
\end{tabular}

If $N=M$, we write $\Theta$ instead of $\Theta_{M}$.

\section{Proof of Proposition 3.5}

1. This is clear.

2. The line of slope $\theta$ does not contain couples $(l, h)$ with $l, h \in \mathbb{Q}^{\times}$; thus $f_{\mathcal{C}(] 0, \theta[)}^{Z}(\underline{U})+f_{\mathcal{C}(] \theta, 2 / \mathbf{t}\left(\theta^{-1}\right)[)}^{Z}(\underline{U})=f_{\mathcal{C}(] 0,2 / \mathbf{t}\left(\theta^{-1}\right)[)}^{Z}(\underline{U})$.

3. In general, $f_{N}$ (respectively $f_{N}^{+}, \Theta_{N}$ ) is a subseries of $f$ (respectively $f^{+}, \Theta$ ); we have already described the domains of convergence of $f, f^{+}$; the domain of convergence of $\Theta$ is $\mathcal{D}^{+}$because $\mathcal{D}^{+} \subset \mathcal{D}$. The assertion on the simultaneous convergence is obvious.

4. By the previous point, $\Theta_{N}(u, v)$ converges on $\mathcal{D}^{+}$; moreover, the right multiplication by $\mathcal{B}(\beta)$ fixes $\mathfrak{h}$ (since $\beta>0$, the map $(x, y) \mapsto\left(\beta x, \beta^{\prime} y\right)$ fixes $\mathfrak{H}$, and $\left.\mathfrak{H} \cdot \mathfrak{B}^{-1}=\mathfrak{h}\right)$. From Lemma 3.2 with $A=\mathcal{B}(\beta), \Theta_{N}(u, v)$ converges on $\mathcal{D}^{+}$to a rational function of $\mathbb{Q}(\underline{u})$ because the slopes $0,2 / \mathbf{t}\left(\theta^{-1}\right)$ are rational.

5. This follows from Lemmata 3.1 (points 3,4) and 3.4.

6. It is enough to deal with the case $N=M$ only, because for all $\mathcal{C}$, if $Z \subset \mathbb{Z}^{2}$, then $f_{\mathcal{C}}^{Z}$ is a subseries of $f_{\mathcal{C}}^{\mathbb{Z}^{2}}$. Let us write $\phi^{+}=f^{+}\left(\underline{\zeta} \underline{u}^{\beta}\right)$. By point 5 of this 
proposition, if $\left(v, v^{\prime}\right) \in \Sigma_{K}\left(f^{+}\right)$, then $v>v^{\prime}>0$. If $\beta$ is such that $\beta \geq \beta^{\prime}>0$, then $\beta v>\beta^{\prime} v^{\prime}>0$ so that if $\mu=\beta v$ is such that $\left(\mu, \mu^{\prime}\right) \in \Sigma_{K}\left(\phi^{+}\right)$, then $\left(\mu, \mu^{\prime}\right) \in \mathcal{C}(] 0,1[) \cap \Sigma\left(\beta M^{*}\right)$.

Since $\phi^{+}(\underline{u})=f^{+}\left(\underline{\zeta} \underline{u}^{\beta}\right)=f^{+}\left(\left(\underline{\zeta}^{\sharp} \underline{u}\right)^{\beta}\right)$ where $\underline{\zeta}^{\sharp}$ is any torsion point such that $\underline{\zeta}^{\sharp \beta}=\underline{\zeta}$, combining Lemma 3.4 and Lemma 3.1 (points 2, 3, 4), we see that $\phi^{+}$is a subseries of $f^{+}\left(\underline{\zeta}^{\sharp} \underline{u}\right)$, hence converging on $\mathcal{D}^{+}$.

If $\beta \geq-\beta^{\prime}>0$ and if $\mu=\beta v$ is such that $\left(\mu, \mu^{\prime}\right) \in \Sigma_{K}\left(\phi^{+}\right)$, then $\mu>$ $-\mu^{\prime}>0$ and $\left(\mu, \mu^{\prime}\right) \in \mathcal{C}(]-1,0[) \cap \Sigma\left(\beta M^{*}\right)$; the conclusion follows again combining Lemmata $3.1,3.4$ because $\phi^{+}$is in this case a subseries of $f\left(\underline{\zeta}^{\sharp} \underline{u}\right)$, converging on $\mathcal{D}$.

Let us now consider $\phi=f\left(\zeta \underline{u}^{\beta}\right)$. By point 4 of this proposition, if $\left(v, v^{\prime}\right) \in$ $\Sigma_{K}(f)$, then $v>-v^{\prime}>0$. If $\beta \in S \backslash\{0\}$ is such that $\beta \geq \beta^{\prime}>0$, then $\beta v>-\beta^{\prime} v^{\prime}>0$. By writing $\mu=\beta v$ for $\mu$ such that $\left(\mu, \mu^{\prime}\right) \in \Sigma_{K}(\phi)$, we see that $\left(\mu, \mu^{\prime}\right) \in \mathcal{C}(]-1,0[) \cap \Sigma\left(\beta M^{*}\right)$ and $\phi$ converges on $\mathcal{D}$.

If $\beta \geq-\beta^{\prime}>0$ then $\mu=\beta v$ and $\left(\mu, \mu^{\prime}\right) \in \Sigma_{K}(\phi)$ implies that $\left(\mu, \mu^{\prime}\right) \in$ $\mathcal{C}(] 0,1[) \cap \beta M^{*}$. In this case, $\phi$ converges on $\mathcal{D}^{+}$. The table follows from these computations. Moreover, $\mathcal{D} \supset \mathcal{D}^{+}$so that in particular, all the series converge on $\mathcal{D}^{+}$.

Let us write:

$$
\begin{aligned}
K_{+} & =\left\{v \in K \text { such that } v>0 \text { and } v^{\prime}>0\right\}, \\
K_{ \pm} & =\left\{v \in K \text { such that } v>0 \text { and } v^{\prime}<0\right\}, \\
\mathcal{I} & =\left\{v \in K \text { such that } v>-v^{\prime}>0 \text { or } v>v^{\prime}>0\right\} .
\end{aligned}
$$

If $E$ is any subset of $K$, we write:

$$
\begin{aligned}
& E_{+}=E \cap K_{+}, \\
& E_{ \pm}=E \cap K_{ \pm} .
\end{aligned}
$$

Thus, $\mathcal{I}=\mathcal{I}_{ \pm} \cup \mathcal{I}_{+}$, with $\mathcal{I}_{ \pm}=\left\{v \in K\right.$ such that $\left.v>-v^{\prime}>0\right\}$ and $\mathcal{I}_{+}=\{v \in$ $K$ such that $\left.v>v^{\prime}>0\right\}$. By Proposition 3.5 point 5, another way to write the series $f_{N}, f_{N}^{+}$and $\Theta_{N}$ is, for $\underline{u}=\Phi\left(z, z^{\prime}\right)$ :

$$
\begin{aligned}
f_{N}(\underline{u}) & =\sum_{v \in \mathcal{I}_{ \pm} \cap N^{*}} e(\mathbf{t}(v \underline{z})), \\
f_{N}^{+}(\underline{u}) & =\sum_{v \in \mathcal{I}_{+} \cap N^{*}} e(\mathbf{t}(v \underline{z})), \\
\Theta_{N}(\underline{u}) & =\sum_{v \in \mathcal{I}_{\mathcal{I}^{*}} N^{*}} e(\mathbf{t}(v \underline{z})) .
\end{aligned}
$$

3.5.2. The rational series $R_{\alpha, \beta, N}, R_{\alpha, \beta, N}^{+}$

We have introduced three types of series $f_{N}, f_{N}^{+}$and $\Theta_{N}$; we need two more types. 
Let $\alpha \in K, \beta, \gamma \in S \backslash\{0\}$, let us suppose that $\beta>0$, let us write $N=\gamma^{-1} M$ and

$$
\underline{\zeta}=\Phi\left(\alpha, \alpha^{\prime}\right), \quad \underline{\zeta}^{\sharp}=\Phi\left(\alpha / \beta, \alpha^{\prime} / \beta^{\prime}\right),
$$

so that $\underline{\zeta}^{\sharp \beta}=\underline{\zeta}$. If $\beta^{\prime}>0$ we write:

$$
\begin{aligned}
& R_{\alpha, \beta, N}(\underline{u})=f_{\beta^{-1} N}\left(\underline{\zeta}^{\sharp} \underline{u}\right)-f_{N}\left(\underline{\zeta}^{\beta}\right) \\
& R_{\alpha, \beta, N}^{+}(\underline{u})=f_{\beta^{-1} N}^{+}\left(\underline{\zeta}^{\sharp} \underline{u}\right)-f_{N}^{+}\left(\underline{\zeta} \underline{u}^{\beta}\right) .
\end{aligned}
$$

Otherwise $\beta^{\prime}<0$, and we write:

$$
\begin{aligned}
& R_{\alpha, \beta, N}(\underline{u})=f_{\beta^{-1} N}\left(\underline{\zeta}^{\sharp} \underline{u}\right)-f_{N}^{+}\left(\underline{\zeta} \underline{u}^{\beta}\right) \\
& R_{\alpha, \beta, N}^{+}(\underline{u})=f_{\beta^{-1} N}^{+}\left(\underline{\zeta}^{\sharp} \underline{u}\right)-f_{N}\left(\underline{\zeta} \underline{u}^{\beta}\right) .
\end{aligned}
$$

Proposition 3.6. With the notations above, we have the following properties.

1. The $K$-support of $R_{\alpha, \beta, N}$ is contained in $\Sigma\left(M_{ \pm}^{*}\right)$ and the $K$-support of $R_{\alpha, \beta, N}^{+}$ is contained in $\Sigma\left(M_{+}^{*}\right)$.

2. For all $\alpha \in K$, if $\beta \in S$ is such that $\beta \geq\left|\beta^{\prime}\right|>0$, then the series $R_{\alpha, \beta, N}$ converges on $\mathcal{D}$ and the series $R_{\alpha, \beta, N}^{+}$converges on $\mathcal{D}^{+}$.

3. For all $\alpha \in K$, if $\beta \in S$ is such that $\beta \geq\left|\beta^{\prime}\right|>0$ then on their convergence domains, $R_{\alpha, \beta, N}, R_{\alpha, \beta, N}^{+}$define rational functions in $\overline{\mathbb{Q}}(\underline{u})$.

4. If $\beta=\beta^{\prime}$ or if $\beta=-\beta^{\prime}$ then, for all $\alpha \in K, R_{\alpha, \beta, N}(\underline{u})=R_{\alpha, \beta, N}^{+}(\underline{u})=0$.

Proof. 1. It is enough to consider the case $\zeta=\zeta^{\sharp}=\underline{1}$. First of all, let us notice that $\Sigma_{K}\left(f_{\beta^{-1} N}\right) \subset \Sigma\left(M_{ \pm}^{*}\right)$ and $\Sigma_{K}\left(f_{\beta^{-1} N}^{+}\right) \subset \Sigma\left(M_{+}^{*}\right)$. Since $\beta>0$, we have $\beta M_{+}^{*} \cup$ $\beta M_{ \pm}^{*} \subset M_{+}^{*} \cup M_{ \pm}^{*}$. If $\beta^{\prime}>0$ then $\beta M_{+}^{*} \subset M_{+}^{*}$ and $\beta M_{ \pm}^{*} \subset M_{ \pm}^{*}$, if $\beta^{\prime}<0$ then $\beta M_{+}^{*} \subset M_{ \pm}^{*}$ and $\beta M_{ \pm}^{*} \subset M_{+}^{*}$. Hence, if $\beta^{\prime}>0$, then $f_{N}\left(\underline{u}^{\beta}\right)$ has its $K$-support contained in $\Sigma\left(M_{ \pm}^{*}\right)$. The same happens to $f_{N}^{+}\left(\underline{u}^{\beta}\right)$ when $\beta^{\prime}<0$. Similarly, if $\beta^{\prime}<0$ then $\Sigma_{K}\left(f_{N}\left(\underline{u}^{\beta}\right)\right) \subset \Sigma\left(M_{+}^{*}\right)$ and if $\beta^{\prime}>0$ then $\Sigma_{K}\left(f_{N}^{+}\left(\underline{u}^{\beta}\right)\right) \subset \Sigma\left(M_{+}^{*}\right)$.

Thus, for all $\beta>0$ and for all $\alpha, R_{\alpha, \beta, N}$ has its $K$-support in $\Sigma\left(M_{ \pm}^{*}\right)$ and $R_{\alpha, \beta, N}^{+}$has its $K$-support in $\Sigma\left(M_{+}^{*}\right)$, because $R_{\alpha, \beta, N}$ (respectively $R_{\alpha, \beta, N}^{+}$) is a linear combination of two series having their $K$-support in $\Sigma\left(M_{ \pm}^{*}\right)$ (respectively in $\left.\Sigma\left(M_{+}^{*}\right)\right)$.

2. The series $f_{\beta^{-1} N}\left(\underline{\zeta}^{\sharp} \underline{u}\right)$ converges on $\mathcal{D}$ and the series $f_{\beta^{-1} N}^{+}\left(\underline{\zeta}^{\sharp} \underline{u}\right)$ converges on $\mathcal{D}^{+}$. Domains of convergence of the series $f_{N}\left(\underline{\zeta} \underline{u}^{\beta}\right)$ and $f_{N}^{+}\left(\underline{\zeta} \underline{u}^{\beta}\right)$ are described in the table of point 5 of Proposition 3.5, depending on the sign of $\beta^{\prime}$. From this, one easily verifies the required properties. 
3. We recall that $\left(\beta^{-1} N\right)^{*}=\beta N^{*}$; let us suppose that $\beta \geq \beta^{\prime}>0$. With the notations introduced above:

$$
\begin{aligned}
& R_{\alpha, \beta, N}\left(\Phi\left(z, z^{\prime}\right)\right)= \\
& =\sum_{\nu \in \beta N^{*} \cap \mathcal{I}_{ \pm}} e\left(\mathbf{t}\left(v\left(\frac{\alpha}{\beta}+\underline{z}\right)\right)\right)-\sum_{\nu \in N^{*} \cap \mathcal{I}_{ \pm}} e(\mathbf{t}(v(\alpha+\beta \underline{z}))) \\
& =\sum_{v \in \beta N^{*} \cap \mathcal{I}_{ \pm}} e\left(\mathbf{t}\left(v\left(\frac{\alpha}{\beta}+\underline{z}\right)\right)\right)-\sum_{\substack{\mu \in \beta N^{*} \\
\beta^{\prime} \mu>-\beta \mu^{\prime}>0}} e\left(\mathbf{t}\left(\mu\left(\frac{\alpha}{\beta}+\underline{z}\right)\right)\right) \\
& =\sum_{\substack{\nu \in \beta N^{*} \\
0<-\nu^{\prime}<\nu \leq-\left(\beta / \beta^{\prime} v^{\prime}\right.}} e\left(\mathbf{t}\left(v\left(\frac{\alpha}{\beta}+\underline{z}\right)\right)\right) \\
& =\sum_{\substack{\left.\left.\left(v, v^{\prime}\right) \in \mathcal{C}(]-1,-\beta^{\prime} / \beta\right]\right) \cap \Sigma\left(\beta N^{*}\right) \\
\beta^{-1} N}} e\left(\mathbf{t}\left(v\left(\frac{\alpha}{\beta}+\underline{z}\right)\right)\right) \\
& =F_{\left.\left.\mathcal{C}(]-1,-\beta^{\prime} / \beta\right]\right)}\left(\frac{\alpha}{\beta}+\underline{z}\right)
\end{aligned}
$$

By Lemma 3.4, we have

$$
R_{0, \beta, N}(\underline{u})=f_{\mathcal{C}}^{Z \cdot \mathcal{B}(\beta)}(\underline{u}),
$$

where $\left.\left.\mathcal{C}=\mathcal{C}(]-1,-\beta^{\prime} / \beta\right]\right) \cdot \mathfrak{B}^{-1}$ and $Z=\mathbb{Z}^{2} \cdot \mathcal{B}(\gamma)$. Lemma 3.1 point 4 implies that $\left.\left.\mathcal{C}=\mathcal{C}(] \rho(-1), \rho\left(-\beta^{\prime} / \beta\right)\right]\right)$. We have already computed $\rho(-1)=0$ (point 3 of Lemma 3.1). Now, we see that:

$$
\begin{aligned}
\rho\left(-\frac{\beta^{\prime}}{\beta}\right) & =\frac{1-\frac{\beta^{\prime}}{\beta}}{\theta^{-1}-\theta^{\prime-1} \frac{\beta^{\prime}}{\beta}} \\
& =\frac{\beta-\beta^{\prime}}{\beta \theta^{-1}-\beta^{\prime} \theta^{\prime-1}} \\
& =\frac{\mathbf{t}\left(\beta \delta^{-1}\right)}{\mathbf{t}\left(\theta^{-1} \beta \delta^{-1}\right)} \in \mathbb{Q} .
\end{aligned}
$$

Thus, the extremities of the interval $\left.] \rho(-1), \rho\left(-\beta^{\prime} / \beta\right)\right]$ are rational. By Lemma 3.2 , we see that $R_{0, \beta, N}$ converges on $\mathcal{D}$ to a rational function in $\mathbb{Q}(u, v)$ (the halflines $l_{1}, l_{2}$ lie in $\mathfrak{h}$ because they are images under right multiplication by $\mathfrak{B}^{-1}$ of half-lines which lie in $\mathfrak{H}$ ).

For $\alpha \in K$, we see that:

$$
R_{\alpha, \beta, N}(\underline{u})=R_{0, \beta, N}\left(\Phi\left(\frac{\alpha}{\beta}, \frac{\alpha^{\prime}}{\beta^{\prime}}\right) \underline{u}\right) .
$$


The coordinates of $\Phi\left(\alpha / \beta, \alpha^{\prime} / \beta^{\prime}\right) \in \mathbb{T}$ are roots of unity. Thus, $R_{\alpha, \beta, N}(\underline{u})$ belongs to $\overline{\mathbb{Q}}(\underline{u})$ (the coefficients of the Taylor expansion at $\underline{0} \in \mathbb{C}^{2}$ lie in some cyclotomic number field of finite degree over $\mathbb{Q}$ ).

So far, we have only considered the case $\beta \geq \beta^{\prime}>0$ and studied the rationality of $R_{\alpha, \beta, N}$. Without giving full details, we sketch the results in the other cases, applying point 4 of Lemma 3.1 (recall that $\underline{u}=\Phi\left(z, z^{\prime}\right)$ ).

$$
\begin{aligned}
& \beta \geq \beta^{\prime}>0, \quad R_{0, \beta, N}^{+}(\underline{u})=F_{\mathcal{C}\left(\left[\beta^{\prime} / \beta, 1\right]\right.}^{\beta^{-1} N}\left(z, z^{\prime}\right)=f_{\mathcal{C}\left(\left[\rho\left(\beta^{\prime} / \beta\right), \rho(1)[)\right.\right.}^{Z \cdot \mathcal{B}(\beta)}, \\
& \beta \geq-\beta^{\prime}>0, R_{0, \beta, N}(\underline{u})=F_{\mathcal{C}\left(\left[-\beta^{\prime} / \beta, 1\right]\right.}^{\beta^{-1} N}\left(z, z^{\prime}\right)=f_{\mathcal{C}\left(\left[\rho\left(-\beta^{\prime} / \beta\right), \rho(1)[)\right.\right.}^{Z \cdot \mathcal{B}(\beta)}, \\
& \beta \geq-\beta^{\prime}>0, R_{0, \beta, N}^{+}(\underline{u})=F_{\left.\left.\mathcal{C}(]-1, \beta^{\prime} / \beta\right]\right)}^{\beta^{-1} N}\left(z, z^{\prime}\right)=f_{\left.\left.\mathcal{C}(] \rho(-1), \rho\left(\beta^{\prime} / \beta\right)\right]\right)}^{Z \cdot \mathcal{B}(\beta)},
\end{aligned}
$$

We have already computed $\rho\left(-\beta^{\prime} / \beta\right)$ and we have seen that it is a rational number. Also $\rho(1)=2 / \mathbf{t}\left(\theta^{-1}\right)$ is a rational number, and

$$
\begin{aligned}
\rho\left(\frac{\beta^{\prime}}{\beta}\right) & =\frac{1+\frac{\beta^{\prime}}{\beta}}{\theta^{-1}+\theta^{\prime-1} \frac{\beta^{\prime}}{\beta}} \\
& =\frac{\beta+\beta^{\prime}}{\theta^{-1} \beta+\theta^{\prime-1} \beta^{\prime}} \\
& =\frac{\mathbf{t}(\beta)}{\mathbf{t}\left(\theta^{-1} \beta\right)}
\end{aligned}
$$

is a rational number too. With the same method as above, we then check that $R_{\alpha, \beta, N}^{+}$ and $R_{\alpha, \beta, N}$ always converge (on $\mathcal{D}$ or $\mathcal{D}^{+}$) to rational functions.

4. If $\beta=\beta^{\prime}$ then ] $\left.-1,-\beta^{\prime} / \beta\right]=\emptyset$ so that $R_{\alpha, \beta, N}=0$ by (3.5). Similarly, $\left[\beta^{\prime} / \beta, 1\left[=\emptyset\right.\right.$ and $R_{\alpha, \beta, N}^{+}=0$ by (3.6).

If $\beta=-\beta^{\prime}$, then $\left[-\beta^{\prime} / \beta, 1[=]-1, \beta^{\prime} / \beta\right]=\emptyset$ so that in this case too, $R_{\alpha, \beta, N}^{+}=R_{\alpha, \beta, N}=0$.

To simplify our notations, we will write $R_{\alpha, \beta}, R_{\alpha, \beta}^{+}$instead of $R_{\alpha, \beta, M}, R_{\alpha, \beta, M}^{+}$. Moreover, if $\alpha=0$, we will drop the corresponding subscript and we will write $R_{\beta}$ instead of $R_{0, \beta}$ and $R_{\beta}^{+}$instead of $R_{0, \beta}^{+}$; similarly, $R_{\beta, N}=R_{0, \beta, N}$ and $R_{\beta, N}^{+}=$ $R_{0, \beta, N}^{+}$.

In Figures 1 and 2 we represent together the $K$-supports of some of the functions introduced so far, and their images under left multiplication by $\mathfrak{B}^{-1}$. 


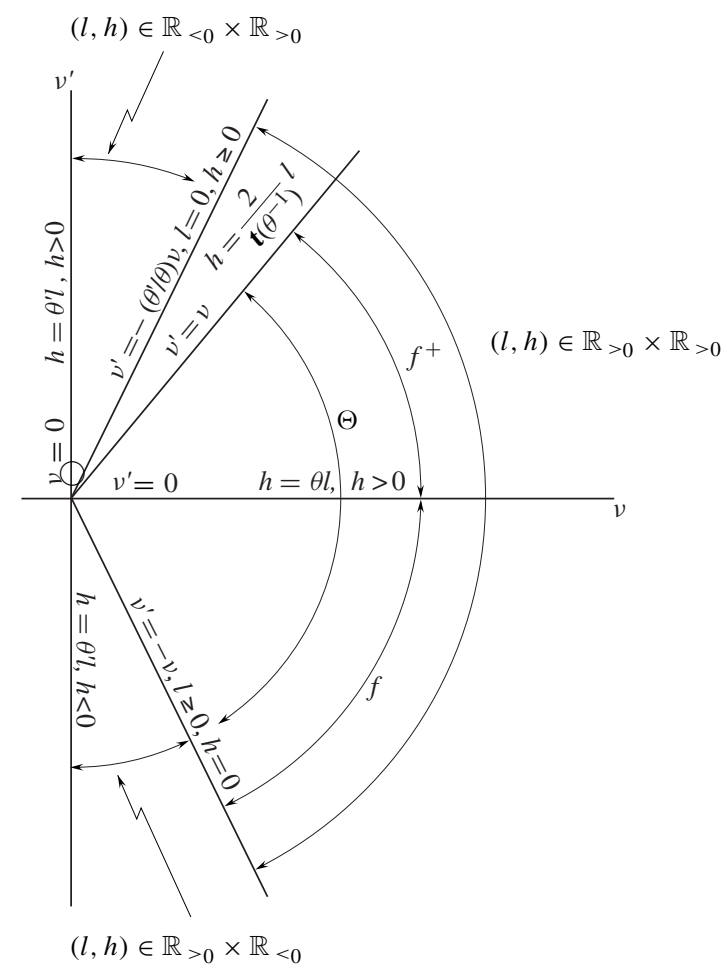

Figure 1.

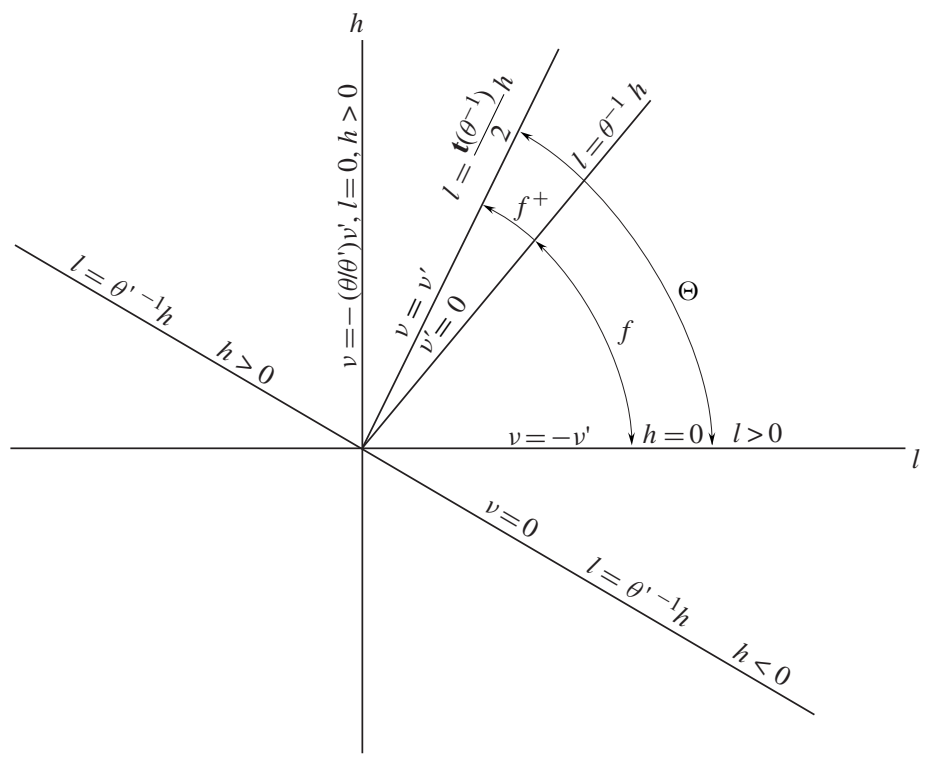

Figure 2. 
Proof of identity (1.12). We notice that if $Z=\left\{(l, h) \in \mathbb{Z}^{2}\right.$ such that $\left.l+h \in 2 \mathbb{Z}\right\}$ then for any sector $\mathcal{C}$ :

$$
f_{\mathcal{C}}^{\mathbb{Z}^{2}}(\underline{U})+f_{\mathcal{C}}^{\mathbb{Z}^{2}}(-\underline{U})=2 f_{\mathcal{C}}^{Z}(\underline{U})
$$

We have already noticed that $\beta:=\sqrt{2} \in S$. In particular $\beta=-\beta^{\prime}$, so that, after Proposition 3.6 point 4 ,

$$
R_{\beta}(\underline{u})=R_{0, \beta, M}(\underline{u})=f_{\beta^{-1} M}^{+}(\underline{u})-f\left(\underline{u}^{\beta}\right)=0 .
$$

Applying (2.4) we see that $\mathcal{B}(\beta)=\left(\begin{array}{cc}1 & 1 \\ 1 & -1\end{array}\right)$ and $Z=\mathbb{Z}^{2} \cdot \mathcal{B}(\beta)$. Thus

$$
f\left(\underline{u}^{\beta}\right)=f_{\beta^{-1} M}^{+}(\underline{u})=f_{\mathcal{C}(] \theta, 2 / \mathbf{t}\left(\theta^{-1}\right)[)}^{Z}(\underline{u}),
$$

and this implies (1.12).

3.5.3. More about the series $R_{\alpha, \beta, N}, R_{\alpha, \beta, N}^{+}$

We give now some applications of Proposition 3.6.

Functional equations. Let $\eta>1$ be a unit of $S$ so that $\eta^{-1} M=M$; clearly, $1>\left|\eta^{\prime}\right|>0$. We recall that $R_{\eta}=R_{0, \eta, M}$ and $R_{\eta}^{+}=R_{0, \eta, M}^{+}$; we have two cases depending on the sign of $\eta^{\prime}$.

First case. $\eta^{\prime}<0$. By definition we have:

$$
\begin{aligned}
f^{+}\left(\underline{U}^{\eta}\right) & =f(\underline{U})-R_{\eta}(\underline{U}), \\
f\left(\underline{U}^{\eta}\right) & =f^{+}(\underline{U})-R_{\eta}^{+}(\underline{U}) .
\end{aligned}
$$

By Proposition 3.5, point $6, f\left(\underline{u}^{\eta}\right)$ converges for $\underline{u} \in \mathcal{D}^{+}$and $f^{+}\left(\underline{u}^{\eta}\right)$ converges for $\underline{u} \in \mathcal{D}$. By Proposition 3.6, point 2, the series $R_{\alpha, \beta, N}$ converges on $\mathcal{D}$ and the series $R_{\alpha, \beta, N}^{+}$converges on $\mathcal{D}^{+}$; more precisely, they converge to rational functions of $\mathbb{Q}(\underline{u})$ (point 3 of Proposition 3.6). The identity (3.7) holds, as an identity of functions, on $\mathcal{D}$, and the identity (3.8) holds, as an identity of functions, on $\mathcal{D}^{+}$.

The multiplicative group $V$ generated by the units $\tilde{\eta} \in S$ such that $\tilde{\eta}>0$ is free and cyclic. There exists one and only one generator $\eta$ with $\eta>1>-\eta^{\prime}>0$; this is the positive fundamental unit of $S$.

If $r>0$ is minimal such that $\theta^{-1}=\left[b_{1}, \ldots, b_{r}, b_{1}, \ldots\right]$ (cf. (1.6)), then

$$
\mathcal{B}(\eta)=\left(\begin{array}{cc}
b_{r} & 1 \\
1 & 0
\end{array}\right) \cdots\left(\begin{array}{cc}
b_{1} & 1 \\
1 & 0
\end{array}\right)
$$

and $\mathcal{B}(\eta)^{2}=\mathcal{B}$ where $\mathcal{B}$ is the matrix of (1.9). 
Second case. $\eta^{\prime}>0$. We have:

$$
\begin{gathered}
f\left(\underline{U}^{\eta}\right)=f(\underline{U})-R_{\eta}(\underline{U}), \\
f^{+}\left(\underline{U^{\eta}}\right)=f^{+}(\underline{U})-R_{\eta}^{+}(\underline{U}) .
\end{gathered}
$$

Again, combining Propositions 3.5 and 3.6 we see that the identity (3.9) holds, as an identity of functions, on $\mathcal{D}$, and the identity (3.10) holds as an identity of functions on $\mathcal{D}^{+}$.

The identity (3.9) for a fixed $\eta \in S_{+}$is one of the functional equations of $f$, that is, one among the relations (1.11) of the introduction. The identity (3.10) is a variant of it, involving the twin series $f^{+}$. The multiplicative group $W$ generated by the units $\tilde{\eta} \in S$ such that $\tilde{\eta}, \tilde{\eta}^{\prime}>0$ is also free and cyclic. There exists one and only one generator $\eta$ with $\eta>1>\eta^{\prime}>0$; this is the totally positive fundamental unit of $S$. If $r>0$ is minimal such that (1.6) holds, we have $\mathcal{B}(\eta)=\mathcal{B}$, the matrix in (1.9).

If there exists a positive unit of $S$ with negative norm, then $V^{2}=W$. If there does not exist a positive unit of $S$ with negative norm, then $V=W$.

Fundamental domains. Let us inspect more closely the relations (3.9) and (3.10) in the second case; let $\eta$ be a unit of $S$ such that $\eta>1>\eta^{\prime}>0$, and $W=\eta^{\mathbb{Z}}$. We choose the fundamental domain for the multiplicative action of $W$ on $K_{ \pm}$

$$
\mathcal{D}(\eta)=\left\{v \in K_{ \pm} \text {such that } 1<-v / v^{\prime} \leq \eta^{2}\right\},
$$

and the fundamental domain for the action of $W$ on $K_{+}$

$$
\mathcal{D}_{+}(\eta)=\left\{v \in K_{+} \text {such that } \eta^{2} \geq v / v^{\prime}>1\right\} .
$$

By the definition of a fundamental domain, if $v \in K_{+}$(respectively $v \in K_{ \pm}$), then there exists one and only one $k \in \mathbb{Z}$ such that $\eta^{k} v \in \mathcal{D}_{+}(\eta)$ (respectively $\left.\eta^{k} v \in \mathcal{D}(\eta)\right)$.

We see that if $k>0$, then

$$
\mathcal{D}\left(\eta^{k}\right)=\bigsqcup_{l=0}^{k-1} \eta^{l} \mathcal{D}(\eta)
$$

where the symbol $\sqcup$ denotes a disjoint union. This implies (identity of formal series):

$$
R_{\eta^{k}, N}(\underline{U})=\sum_{l=0}^{k-1} R_{\eta, N}\left(\underline{U}^{\eta^{l}}\right),
$$

where $N=\beta^{-1} M, \beta \in S \backslash\{0\}$. In particular, since $\mathcal{I}_{ \pm}=\lim _{k \rightarrow \infty} \mathcal{D}\left(\eta^{k}\right)$ :

$$
f(\underline{U})=\sum_{n=0}^{\infty} R_{\eta, N}\left(\underline{U}^{\eta^{n}}\right)=\lim _{k \rightarrow \infty} R_{\eta^{k}, N}(\underline{U}) .
$$


Let us explain this identity. In general, infinite sums (or limits) are not allowed in $\mathbb{C}\{\underline{U}\}$, but for all $h \neq k$, the $K$-supports of $R_{\eta, N}\left(\underline{U}^{\eta^{h}}\right)$ and $R_{\eta, N}\left(\underline{U}^{\eta^{k}}\right)$ are disjoint $\left(\mathcal{D}(\eta), \mathcal{D}_{+}(\eta)\right.$ are fundamental domains), so that the right-hand side of (3.11) above is a well defined element of $\mathbb{C}\{\underline{U}\}$.

We also have

$$
\Sigma_{K}\left(f_{N}\right)=\Sigma\left(\mathcal{I}_{ \pm} \cap N^{*}\right)=\bigsqcup_{l=0}^{\infty} \Sigma\left(\eta^{l}\left(\mathcal{D}(\eta) \cap N^{*}\right)\right)
$$

In a similar way, $\mathcal{D}_{+}\left(\eta^{k}\right)$ is equal to the disjoint union $\sqcup_{l=0}^{k-1} \eta^{l} \mathcal{D}_{+}(\eta)$ and $\mathcal{I}_{+}=$ $\lim _{n \rightarrow \infty} \mathcal{D}_{+}\left(\eta^{n}\right)$, so that:

$$
f_{N}^{+}(\underline{U})=\sum_{n=0}^{\infty} R_{\eta, N}^{+}\left(\underline{U}^{\eta^{n}}\right)=\lim _{k \rightarrow \infty} R_{\eta^{k}, N}^{+}(\underline{U}) .
$$

The latter equality in $\mathbb{C}\{\underline{U}\}$ is equivalent to

$$
\Sigma_{K}\left(f_{N}^{+}\right)=\Sigma\left(\mathcal{I}_{+} \cap N^{*}\right)=\bigsqcup_{l=0}^{\infty} \Sigma\left(\eta^{l}\left(\mathcal{D}_{+}(\eta) \cap N^{*}\right)\right)
$$

The equalities (3.11) and (3.13) hold in $\mathbb{C}\{\underline{U}\}$, but it is easy to show that they also hold as equalities of convergent series, respectively for $\underline{u} \in \mathcal{D}$ and for $\underline{u} \in \mathcal{D}^{+}$.

\section{Linear independence over $\mathbb{Q}$ of formal series}

If $M_{2} \subset M_{1}$ are complete modules, we denote by $\left[M_{1}: M_{2}\right]$ the index of $M_{2}$ in $M_{1}$. We start with two elementary lemmata.

Lemma 4.1 (Duality). Let $M_{2} \subset M_{1}$ be two complete modules of $K$, let $v \in M_{2}^{*}$. We have:

$$
\sum_{\mu \in M_{1} / M_{2}} e(\mathbf{t}(\mu \nu))=\left\{\begin{array}{l}
0 \text { if } v \notin M_{1}^{*}, \\
{\left[M_{1}: M_{2}\right] \text { if } v \in M_{1}^{*},}
\end{array}\right.
$$

the sum being indexed by a complete set of representatives of $M_{1}$ non-equivalent modulo $\mathrm{M}_{2}$.

Proof. The sums in (4.1) are well defined and do not depend on the representatives chosen in any class.

By the theory of elementary divisors, one can choose $\alpha, \beta \in K$ such that $M_{1}=\alpha \mathbb{Z}+\beta \mathbb{Z}$ and $M_{2}=a \alpha \mathbb{Z}+b \beta \mathbb{Z}$ with positive integers $a, b$. With a dual basis $M_{1}^{*}=\alpha^{*} \mathbb{Z}+\beta^{*} \mathbb{Z}$ one gets a dual basis $M_{2}^{*}=\left(\alpha^{*} / a\right) \mathbb{Z}+\left(\beta^{*} / b\right) \mathbb{Z}$. Let us 
consider $v=c \alpha^{*} / a+d \beta^{*} / b \in M_{2}^{*}(c, d \in \mathbb{Z})$. We have:

$$
\begin{aligned}
\sum_{\mu \in M_{1} / M_{2}} e(\mathbf{t}(\mu \nu)) & =\sum_{l=0}^{a-1} \sum_{h=0}^{b-1} e\left(\mathbf{t}\left((l \alpha+h \beta)\left(c \alpha^{*} / a+d \beta^{*} / b\right)\right)\right) \\
& =\sum_{l=0}^{a-1} e(l c / a) \sum_{h=0}^{b-1} e(h d / b)
\end{aligned}
$$

If $v \in M_{2}^{*} \backslash M_{1}^{*}$ then $a$ does not divide $c$ or $b$ does not divide $d$, and the latter product is zero. Otherwise, $v \in M_{1}^{*}$, and the sum is $a b$, but $a b$ is just the index $\left[M_{1}: M_{2}\right]$.

Lemma 4.2 (Vandermonde matrices). Let $L>0$ be a rational integer, let us choose an ordering of complete sets of non-equivalent representatives of $M^{*} / L M^{*}$ and $L^{-1} M / M$. Then, the matrix $\mathcal{M}(L)$ below is non-singular:

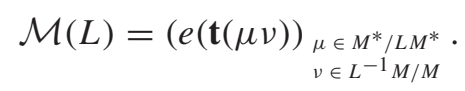

Proof. The matrix is clearly well defined. Let us choose a $\mathbb{Z}$-basis $\left(\mu_{1}, \mu_{2}\right)$ of $M$, let $\left(\mu_{1}^{*}, \mu_{2}^{*}\right)$ be the dual $\mathbb{Z}$-basis of $M^{*}$.

We have, up to permutations of rows and columns:

$$
\begin{aligned}
\mathcal{M}(L) & =\left(e\left(\mathbf{t}\left(\left(a_{1} \mu_{1}+a_{2} \mu_{2}\right)\left(b_{1} \mu_{1}^{*}+b_{2} \mu_{2}^{*}\right)\right) / L\right\}\right)_{\left(a_{1}, a_{2}\right),\left(b_{1}, b_{2}\right)} \\
& =\left(e\left(\left(a_{1} b_{1}+a_{2} b_{2}\right) / L\right)\right)_{\left(a_{1}, a_{2}\right),\left(b_{1}, b_{2}\right)},
\end{aligned}
$$

where the rows are indexed by the couples $\left(a_{1}, a_{2}\right) \in \mathbb{Z}^{2}$ with $0 \leq a_{1}, a_{2} \leq L-1$ and the columns are indexed by the couples $\left(b_{1}, b_{2}\right) \in \mathbb{Z}^{2}$ with $0 \leq b_{1}, b_{2} \leq L-1$.

Thus the matrix $\mathcal{M}(L)$ is, up to permutations of rows and columns, the Kronecker square of the Vandermonde matrix:

$$
(e(a b / L))_{0 \leq a, b \leq L-1} .
$$

Let $D$ be the determinant of this matrix. We have $\operatorname{det}(\mathcal{M}(L))=D^{2 L}$; but $D$ is non-zero and the matrix $\mathcal{M}(L)$ is non-singular.

Lemma 4.3. Let us consider elements $\alpha_{1}, \ldots, \alpha_{m} \in K, \beta_{1}, \ldots, \beta_{m} \in S \backslash\{0\}$, let us denote by $N_{i}$ the complete module $\beta_{i}^{-1} M$, let $\mathcal{F}$ be a sector with non-empty interior. Let us denote by $\mathcal{V}$ the $\mathbb{C}$-vector space spanned by the $m$-tuples $\left(c_{1}, \ldots, c_{m}\right) \in \mathbb{C}^{m}$ such that

$$
\sum_{i=1}^{m} c_{i} F_{\mathcal{F}}^{N_{i}}\left(\frac{\alpha_{i}}{\beta_{i}}+Z, \frac{\alpha_{i}^{\prime}}{\beta_{i}^{\prime}}+Z^{\prime}\right)=0 .
$$


Let us write, for $i=1, \ldots, m$ and $v \in M^{*}$,

$$
A_{v, i}= \begin{cases}e\left(\mathbf{t}\left(\alpha_{i} v / \beta_{i}\right)\right) & \text { if } v \in N_{i}^{*}, \\ 0 & \text { otherwise. }\end{cases}
$$

Let $\ell_{1}, \ldots, \ell_{m} \in \mathbb{N} \backslash\{0\}$ be such that $\ell_{i} \alpha_{i} \in M(i=1, \ldots, m)$, let us choose integers $g_{1}, \ldots, g_{m} \in \mathbb{N} \backslash\{0\}$ such that $g_{i} \beta_{i}^{-1} \in S(i=1, \ldots, m)$. Let us write:

$$
L=\text { least common multiple of } g_{1} \ell_{1}, \ldots, g_{m} \ell_{m} \text {. }
$$

Then, for all $i=1, \ldots, m$ we have $L M^{*} \subset N_{i}^{*}$, and for any complete set $\mathcal{G}$ of non-equivalent representatives of $M^{*}$ modulo $L M^{*}, \mathcal{V}$ is the kernel of the left multiplication by $\mathcal{M}(L) \cdot \mathcal{N}(L)$, where

$$
\mathcal{N}(L)=\left(A_{v, i}\right)_{\substack{v \in \mathcal{G} \\ 1 \leq i \leq m}} .
$$

Proof. Let us recall that given a complete module $N$, if $\alpha \in K^{\times}$, then $\ell \alpha \in N$ for some $\ell \in \mathbb{N} \backslash\{0\}$ (this was used in Lemma 2.1). The numbers $g_{1}, \ldots, g_{m}$ exist because $S$ is a complete module (cf. proof of Lemma 2.1).

Let $\left(c_{1}, \ldots, c_{m}\right)$ be any element of $\mathcal{V}$. The relation (4.2) is equivalent to infinitely many linear relations indexed by the elements $v \in M^{*}$ such that $\left(v, v^{\prime}\right) \in \mathcal{F}$, and involving the coefficients $c_{i}$ for $i=1, \ldots, m$. These relations are:

$$
\sum_{\substack{i \text { such that } \\\left(\nu, v^{\prime}\right) \in \mathcal{F} \cap N_{i}^{*}}} c_{i} e\left(\mathbf{t}\left(\alpha_{i} v / \beta_{i}\right)\right)=0, \quad v \in M^{*} .
$$

However, in (4.4) almost all relations are redundant; let us explain this fact. Let

$$
\mathcal{N}=\left(A_{v, i}\right)
$$

be the matrix of these relations (its rows are in a one-to-one correspondence with the elements $v \in M^{*}$ such that $\left(v, v^{\prime}\right) \in \mathcal{F}$, its columns are indexed by $\left.i=1, \ldots, m\right)$.

Let us recall the equality $S(N)=S\left(N^{*}\right)$. Since $g_{i} \beta_{i}^{-1} \in S$ for all $i$ we have $g_{i} \beta_{i}^{-1} M^{*} \subseteq M^{*}$ and $g_{i} M^{*} \subseteq \beta_{i} M^{*}$; thus, for all $i=1, \ldots, m, L M^{*} \subseteq g_{i} M^{*} \subseteq$ $N_{i}^{*}=\beta_{i} M^{*}$; notice also that $\left[M^{*}: L M^{*}\right]=L^{2}$.

The matrix $\mathcal{N}(L)$ does not depend on the choice of $\mathcal{G}$. Indeed, if $\lambda \in L M^{*}$ (so that $\lambda=L \mu$ with $\mu \in M^{*}$ ) then, for all $i$,

$$
\mathbf{t}\left(\frac{\lambda \alpha_{i}}{\beta_{i}}\right)=\frac{L}{\ell_{i} g_{i}} \mathbf{t}\left(\ell_{i} \alpha_{i} \mu \frac{g_{i}}{\beta_{i}}\right) \in \mathbb{Z}
$$

because $L /\left(\ell_{i} g_{i}\right) \in \mathbb{Z}, \ell_{i} \alpha_{i} \in M$ and $g_{i} / \beta_{i} \in S$, so that $\mu g_{i} / \beta_{i} \in M^{*}$. Thus if $v \in$ $M^{*}$, then $\mathbf{t}\left(\alpha_{i}(v+\lambda) / \beta_{i}\right) \in \mathbf{t}\left(\alpha_{i} v / \beta_{i}\right)+\mathbb{Z}$ and $e\left(\mathbf{t}\left(\alpha_{i}(v+\lambda) / \beta_{i}\right)\right)=e\left(\mathbf{t}\left(\alpha_{i} v / \beta_{i}\right)\right)$. 
We claim that there exists a complete set $\mathcal{G}$ of non-equivalent representatives of $M^{*}$ modulo $L M^{*}$ such that $\mathcal{F}$ contains $\Sigma(\mathcal{G})$. Indeed, given any complete set $\tilde{\mathcal{G}}$ of nonequivalent representatives of $M^{*}$ modulo $L M^{*}$, there exists a bounded subset $\mathcal{K}$ of $\mathbb{R}^{2}$ such that $\Sigma(\tilde{\mathcal{G}}) \subset \mathcal{K}$. Now, for any $\mu \in M^{*}$, the translated $\mu+\tilde{\mathcal{G}}$ is also a set of non-equivalent representatives of $M^{*}$ modulo $L M^{*}$, and $\Sigma(\mu+\tilde{\mathcal{G}}) \subset\left(\mu, \mu^{\prime}\right)+\mathcal{K}$. It is certainly possible to find $\mu \in M^{*}$ such that $\left(\mu, \mu^{\prime}\right)+\mathcal{K} \subset \mathcal{F}$ (because $\Sigma\left(M^{*}\right)$ is a lattice of $\mathbb{R}^{2}$ and $\mathcal{F}$ is a sector) and this suffices to prove the claim, by setting $\mathcal{G}=\mu+\tilde{\mathcal{G}}$.

Let us consider, to construct our matrix $\mathcal{N}(L), \mathcal{G}$ a complete set of representatives of $M^{*}$ modulo $L M^{*}$ such that $\Sigma(\mathcal{G}) \subset \mathcal{F}$.

Since $\mathcal{F}$ contains $\Sigma(\mathcal{G})$ the matrix $\mathcal{N}$ is equal, up to a permutation of its rows, to a block matrix made by an infinite column whose entries are copies of $\mathcal{N}(L)$. With $\underline{c}=\left(c_{1}, \ldots, c_{m}\right)$, the identities (4.4) are equivalent to:

$$
\mathcal{N}(L) \cdot{ }^{\mathrm{t}} \underline{c}={ }^{\mathrm{t}} \underline{\mathrm{in}} \mathbb{C}^{L^{2}},
$$

so that $\mathcal{V}$ is the kernel of the left multiplication by $\mathcal{N}(L)$. But $\mathcal{M}(L)$ is nonsingular (Lemma 4.2), thus $\mathcal{V}$ is also the kernel of the left multiplication by $\mathcal{M}(L)$. $\mathcal{N}(L)$. series:

Let $\underline{U}=(U, V)$ be a couple of indeterminates. We consider again the formal

$$
\mathbb{F}(\underline{U}):=f_{\mathbb{R}^{2} \backslash\{(0,0)\}}^{\mathbb{Z}^{2}}(\underline{U})=\sum_{\substack{h, l \in \mathbb{Z} \\(h, l) \neq(0,0)}} U^{l} V^{h} \in \mathbb{C}\{\underline{U}\} .
$$

Proposition 4.4. Let us consider elements $\alpha_{1}, \ldots, \alpha_{m} \in K, \beta_{1}, \ldots, \beta_{m} \in S \backslash\{0\}$, let us denote by $N_{i}$ the complete module $\beta_{i}^{-1} M(i=1, \ldots, m)$, let us write

$$
\underline{\zeta}_{i}=\Phi\left(\alpha_{i}, \alpha_{i}^{\prime}\right), \quad i=1, \ldots, m,
$$

let $\mathcal{F}$ be a sector with non-empty interior. Then, the following conditions are equivalent.

1. The formal double Laurent series

$$
\mathbb{F}\left(\underline{\zeta}_{i} \underline{U}^{\beta_{i}}\right), \quad i=1, \ldots, m
$$

are $\mathbb{Q}$-linearly dependent.

2. The formal Fourier series

$$
F_{\mathcal{F}}^{N_{i}}\left(\frac{\alpha_{i}}{\beta_{i}}+Z, \frac{\alpha_{i}^{\prime}}{\beta_{i}^{\prime}}+Z^{\prime}\right), \quad i=1, \ldots, m
$$

are $\mathbb{C}$-linearly dependent. 
3. The formal Fourier series

$$
F_{\mathcal{F}}^{N_{i}}\left(\frac{\alpha_{i}}{\beta_{i}}+Z, \frac{\alpha_{i}^{\prime}}{\beta_{i}^{\prime}}+Z^{\prime}\right), \quad i=1, \ldots, m
$$

are $\mathbb{Q}$-linearly dependent.

Proof. Let us write

$$
\underline{\zeta}_{i}^{\sharp}=\Phi\left(\frac{\alpha_{i}}{\beta_{i}}, \frac{\alpha_{i}^{\prime}}{\beta_{i}^{\prime}}\right), \quad i=1, \ldots, m
$$

so that $\underline{\zeta}_{i}^{\sharp} \beta_{i}=\underline{\zeta}_{i}$ for all $i$. Let us consider $i \in\{1, \ldots, m\}$ and $\mu \in M^{*}$. Since

$$
M\left(\underline{\zeta}_{i} \underline{U}^{\beta_{i}}\right)^{\mu}=M\left(\underline{\zeta}_{i}^{\sharp \beta_{i}} \underline{U}^{\beta_{i}}\right)^{\mu}=M\left(\underline{\zeta}_{i}^{\sharp} \underline{U}^{\mu \beta_{i}}\right.
$$

we have, applying Lemma 3.4 with $\Phi\left(Z, Z^{\prime}\right)=\underline{U}$ :

$$
\begin{aligned}
\mathbb{F}\left(\underline{\zeta}_{i} \underline{U}^{\beta_{i}}\right) & =f_{\mathbb{R}^{2} \backslash\{(0,0)\}}^{\mathbb{Z}^{2}}\left(\underline{\zeta}_{i} \underline{U}^{\beta_{i}}\right) \\
& \left.=f_{\mathbb{R}^{2} \backslash\{(0,0)\}}^{\mathbb{Z}^{2} \mathcal{B}\left(\beta_{i}\right)} \underline{\zeta}_{i}^{\sharp} \underline{U}\right) \\
& =F_{\mathbb{R}^{2} \backslash\{(0,0)\}}^{N_{i}}\left(\frac{\alpha_{i}}{\beta_{i}}+Z, \frac{\alpha_{i}^{\prime}}{\beta_{i}^{\prime}}+Z^{\prime}\right),
\end{aligned}
$$

and $F_{\mathcal{F}}^{N_{i}}\left(\left(\alpha_{i} / \beta_{i}\right)+Z,\left(\alpha_{i}^{\prime} / \beta_{i}^{\prime}\right)+Z^{\prime}\right)$ is a subseries of the series in (4.7). Thus for all $i$, the series in the second condition of the proposition can be viewed as a subseries of the series in the first condition, with $\Phi\left(Z, Z^{\prime}\right)=\underline{U}$, and the second condition follows from the first.

We now prove that the second condition implies the third condition; by using Lemma 4.3, we want to show that $\mathcal{V}$ is defined over $\mathbb{Q}$. Let $L$ be defined by (4.3).

Let us write:

$$
\mathcal{M}(L) \cdot \mathcal{N}(L)=\left(B_{\mu, i}\right)_{\substack{\mu \in L^{-1} M / M \\ 1 \leq i \leq m}}
$$

We notice that if $\mu \in L^{-1} M / M$, then $\mu+\alpha_{i} / \beta_{i} \in L^{-1} M / M(i=1, \ldots, m)$; Lemma 4.1 can be applied to get the equalities:

$$
B_{\mu, i}=\left\{\begin{array}{l}
{\left[\beta_{i} M^{*}: L M^{*}\right] \text { if } \mu+\alpha_{i} / \beta_{i} \in \beta_{i}^{-1} M} \\
0 \text { if } \mu+\alpha_{i} / \beta_{i} \notin \beta_{i}^{-1} M
\end{array}\right.
$$

because

$$
\begin{aligned}
B_{\mu, i} & =\sum_{v \in \beta_{i} M^{*} / L M^{*}} e(\mathbf{t}(v \mu)) A_{\beta, i} \\
& =\sum_{\nu \in \beta_{i} M^{*} / L M^{*}} e\left(\mathbf{t}\left(v\left(\mu+\alpha_{i} / \beta_{i}\right)\right)\right) .
\end{aligned}
$$


Hence, the matrix $\mathcal{M}(L) \cdot \mathcal{N}(L)$ has rational integer entries. Since $\mathcal{V}$ is the kernel of the left multiplication by $\mathcal{M}(L) \cdot \mathcal{N}(L)$, it is defined over $\mathbb{Q}$. By hypothesis, $\mathcal{V} \neq\{\underline{0}\}$; this suffices to obtain the third condition from the second.

We now prove that the third condition implies the first. Let $\mathcal{Z}$ be the vector space of the $m$-tuples $\left(c_{1}, \ldots, c_{m}\right) \in \mathbb{C}^{m}$ such that

$$
\sum_{i=1}^{m} c_{i} F_{\mathbb{R}^{2} \backslash\{(0,0)\}}^{N_{i}}\left(\frac{\alpha_{i}}{\beta_{i}}+Z, \frac{\alpha_{i}^{\prime}}{\beta_{i}^{\prime}}+Z^{\prime}\right)=0 .
$$

Since $\mathbb{R}^{2} \backslash\{(0,0)\}$ is a sector, Lemma 4.3 applies and says that $\mathcal{Z}$ is also the kernel of the left multiplication by $\mathcal{M}(L) \cdot \mathcal{N}(L)$ ( $L$ being defined in (4.3)).

Thus, $\mathcal{Z}=\mathcal{V}$ and $\mathcal{Z} \cap \mathbb{Q}^{m}=\mathcal{V} \cap \mathbb{Q}^{m}$; the conclusion follows from (4.7).

\subsection{Further properties of the series $\mathbb{F}$.}

Let $\Omega \in \mathbb{C}\{\underline{U}\}, \Omega(\underline{U})=\sum_{l, h} c_{l, h} U^{l} V^{h}$. The complex conjugate $\bar{\Omega}$ of $\Omega$ is the formal series

$$
\bar{\Omega}(\underline{U})=\sum_{l, h} \overline{c_{l, h}} U^{l} V^{h} \in \mathbb{C}\{\underline{U}\},
$$

where ${ }^{-}$denotes the complex conjugation in $\mathbb{C}$.

Proposition 4.5. Let $\beta$ be in $S \backslash\{0\}$ and $\underline{\zeta} \in \mathbb{T}$ be a torsion point. We have the following properties.

1. If $\eta$ is a unit of $S$ such that $\underline{\zeta}^{\eta}=\underline{\zeta}$, then

$$
\mathbb{F}\left(\underline{\zeta}^{\eta \beta}\right)=\mathbb{F}\left(\underline{\zeta} \underline{U}^{\beta}\right)
$$

2. In $\mathbb{C}\{\underline{U}\}$ we have

$$
\overline{\mathbb{F}\left(\underline{\zeta} \underline{U}^{\beta}\right)}=\mathbb{F}\left(\underline{\zeta}^{-1} \underline{U}^{\beta}\right) .
$$

In particular, if $\underline{\zeta}_{1}, \ldots, \underline{\zeta}_{m}$ are torsion points, $\eta_{1}, \ldots, \eta_{m}$ are units of $S$ such that $\underline{\zeta}_{i}^{\eta_{i}}=\underline{\zeta}_{i}$ for all $i$, and $\beta_{1}, \ldots, \beta_{m}$ are elements of $S \backslash\{0\}$, then in $\mathbb{C}\{\underline{U}\}$ and for $\bar{i}=1, \ldots, m$, the series $\mathbb{F}\left(\underline{\zeta}_{i}^{-1} \underline{U}^{\beta_{i}}\right)$ are $\mathbb{Q}$-linearly independent if and only if the series $\mathbb{F}\left(\underline{\zeta}_{i} \underline{U}^{\beta_{i}}\right)$ are $\mathbb{Q}$-linearly independent, if and only if the series $\mathbb{F}\left(\underline{\zeta}_{i} \underline{U}^{\eta_{i} \beta_{i}}\right)$ are $\mathbb{Q}$-linearly independent.

Proof. 1. Since $\eta\left(M^{*} \backslash\{0\}\right)=M^{*} \backslash\{0\}$, we have $\mathbb{F}\left(\left(\underline{\zeta} \underline{U}^{\beta}\right)^{\eta}\right)=\mathbb{F}\left(\underline{\zeta} \underline{U}^{\beta}\right)$. Now, $\left(\underline{\zeta}^{\beta}\right)^{\eta}=\underline{\zeta} \underline{U}^{\eta \beta}$.

2. By Lemma 2.1, any torsion point $\underline{\zeta} \in \mathbb{T}$ satisfies $\underline{\zeta}=\Phi\left(\alpha, \alpha^{\prime}\right)$ for some $\alpha \in K$. If $\Phi\left(Z, Z^{\prime}\right)=\underline{U}$ then

$$
M\left(\underline{\zeta}^{-1} \underline{U}^{\beta}\right)^{v}=e(\mathbf{t}(v(-\alpha+\beta \underline{Z})))=\overline{e(\mathbf{t}(v \alpha))} M\left(\underline{U}^{\beta}\right)^{v}=\overline{M\left(\underline{\zeta} \underline{U}^{\beta}\right)^{v}}
$$


for all $\beta \in S$ and $\nu \in M^{*}$. Hence

$$
\begin{aligned}
\overline{\mathbb{F}\left(\underline{\zeta} \underline{U}^{\beta}\right)} & =\sum_{v \in M^{*} \backslash\{0\}} \overline{M\left(\underline{\zeta} \underline{U}^{\beta}\right)^{v}} \\
& =\sum_{v \in M^{*} \backslash\{0\}} M\left(\underline{\zeta}^{-1} \underline{U}^{\beta}\right)^{v} \\
& =\mathbb{F}\left(\underline{\zeta}^{-1} \underline{U}^{\beta}\right) .
\end{aligned}
$$

We can conclude the proof; by points 1,2 , since $c_{i} \in \mathbb{Q}$ for all $i$, we have:

$$
\begin{aligned}
0 & =\overline{\sum_{i=1}^{m} c_{i} \mathbb{F}\left(\underline{\zeta}_{i} \underline{U}^{\beta_{i}}\right)} \\
& =\sum_{i=1}^{m} c_{i} \overline{\mathbb{F}\left(\underline{\zeta}_{i} \underline{U}^{\beta_{i}}\right)} \\
& =\sum_{i=1}^{m} c_{i} \mathbb{F}\left(\underline{\zeta}_{i}^{-1} \underline{U}^{\beta_{i}}\right) \\
& =\sum_{i=1}^{m} c_{i} \mathbb{F}\left(\underline{\zeta}_{i}^{-1} \underline{U}^{\eta_{i} \beta_{i}}\right) .
\end{aligned}
$$

\subsection{Proof of one half of our theorem}

Lemma 4.6. Let $\beta \in S \backslash\{0\}$. The map $\mathbb{T} \rightarrow \mathbb{T}$ defined by $\underline{u} \mapsto \underline{u}^{\beta}$ is a group homomorphism whose kernel is a finite subgroup of $\mathbb{T}$ with $|\mathbf{n}(\beta)|=\left|\bar{\beta} \beta^{\prime}\right|$ elements. Let us consider finitely many torsion points $\underline{\zeta}_{1}, \ldots, \underline{\zeta}_{m} \in \mathbb{T}$. There exists an irrational unit $\eta \in S$ with $\eta, \eta^{\prime}>0$ such that $\underline{\zeta}_{i}^{\eta}=\underline{\zeta}_{i}$ for all $i=1, \ldots, m$. $|\mathbf{n}(\beta)|$.

We call the maps $\underline{u} \mapsto \underline{u}^{\beta}$ of the lemma isogenies; the degree of $\underline{u} \mapsto \underline{u}^{\beta}$ is

Proof of Lemma 4.6. The first part is clear, since $\beta^{-1} M / M \cong M^{*} /\left(\beta M^{*}\right)$ is a finite group with $|\mathbf{n}(\beta)|$ elements.

Let $\eta$ be a unit of $S$; from the first part of the lemma we see that the isogeny $\mathbb{T} \rightarrow \mathbb{T}$ defined by $\underline{u} \mapsto \underline{u}^{\eta}$ is an automorphism (its degree is 1 ).

The set $\zeta^{S}=\left\{\zeta^{\beta} ; \bar{\beta} \in S\right\}$ is a finite subgroup of $\mathbb{T}$ and is stable under the action of $S$. The automorphism $\underline{u} \mapsto \underline{u}^{\eta}$ acts as a permutation of $\zeta^{S}$, so that a suitable non-zero integer power of it induces the identity map on $\zeta^{S}$.

Now, given $\underline{\zeta}_{1}, \ldots, \underline{\zeta}_{m}$ as above and an irrational unit $\tilde{\eta} \in S$, there are integers $p_{1}, \ldots, p_{m}>0$ such that $\underline{\zeta}_{i}^{\tilde{\eta}_{i}}=\underline{\zeta}_{i}$ for all $i$. The lemma follows by setting $\eta=\tilde{\eta}^{2 p_{1} \cdots p_{m}}$. 
Lemma 4.7. Let $\underline{u}_{1}, \ldots, \underline{u}_{m} \in \mathbb{T} \cap \mathcal{D}$ and $\underline{v} \in \mathbb{T}$ be such that there exist $\beta_{1}, \ldots, \beta_{m} \in$ $S \backslash\{0\}$ and $\alpha_{1}, \ldots, \alpha_{m} \in K$ satisfying (2.7) (thus the $m$-tuple $\left(\underline{u}_{1}, \ldots, \underline{u}_{m}\right)$ satisfies the rank one hypothesis). Let $W$ be the multiplicative group generated by the units $\eta>0$ with $\eta^{\prime}>0$ such that $\Phi\left(\eta \alpha_{i}, \eta^{\prime} \alpha_{i}^{\prime}\right)=\Phi\left(\alpha_{i}, \alpha_{i}^{\prime}\right)$ for all $i$. Then, there exist $\underline{w}_{1}, \ldots, \underline{w}_{m}, \underline{a} \in \mathbb{T} \cap \mathcal{D}^{+}$, units $\eta^{*}, \tilde{\eta} \in W$, such that $\underline{a}^{\prime} \underline{v}^{\tilde{\eta}}$ or $\underline{a}=\underline{v}^{-\tilde{\eta}}, \underline{w}_{i}=\underline{u}_{i}^{\eta^{*}}$ (for all $i=1, \ldots, m)$ and elements $\gamma_{1}, \ldots, \gamma_{m} \in S$ satisfying

$$
\underline{w}_{i}=\Phi\left(\alpha_{i}, \alpha_{i}^{\prime}\right) \underline{a}^{\gamma_{i}} \text { with } \gamma_{i} \geq\left|\gamma_{i}^{\prime}\right|>0 \quad \text { for all } i=1, \ldots, m \text {. }
$$

The sign of the exponent of $\underline{v}$ in the expression of $\underline{a}$ is + if $\beta_{1}>0$ and - if $\beta_{1}<0$.

Moreover, the series $\mathbb{F}\left(\Phi\left(\alpha_{i}, \alpha_{i}^{\prime}\right) \underline{U}^{\beta_{i}}\right)$ are $\mathbb{Q}$-linearly independent if and only if the series $\mathbb{F}\left(\Phi\left(\alpha_{i}, \alpha_{i}^{\prime}\right) \underline{U}^{\gamma_{i}}\right)$ are $\mathbb{Q}$-linearly independent $(i=1, \ldots, m)$.

Proof. Let $\mathcal{H}$ be the complex upper half-plane

$$
\mathcal{H}=\{z \in \mathbb{C} \text { such that } \Im(z)>0\},
$$

let us introduce two subsets of $\mathbb{C}^{2}$ :

$$
\begin{aligned}
\mathcal{W} & =\left\{\left(z, z^{\prime}\right) \in \mathcal{H} \times \mathbb{C} \text { with } \Im\left(z^{\prime}\right)<\Im(z)\right\}, \\
\mathcal{W}^{+} & =\left\{\left(z, z^{\prime}\right) \in \mathcal{H} \times \mathbb{C} \text { with }-\Im(z)<\Im\left(z^{\prime}\right)<\Im(z)\right\} .
\end{aligned}
$$

Clearly $\mathcal{W}^{+} \subset \mathcal{W}$. Moreover, by using (1.2) and some of the identities (with $\left.(u, v)=\Phi\left(z, z^{\prime}\right)\right)$ :

$$
\begin{aligned}
|u| & =\exp \left\{-2 \pi \delta^{-1}\left(\Im(z)-\Im\left(z^{\prime}\right)\right)\right\} \\
|u||v|^{\theta} & =\exp \{-2 \pi \theta \Im(z)\} \\
|u \| v|^{\theta^{\prime}} & =\exp \left\{-2 \pi \theta^{\prime} \Im\left(z^{\prime}\right)\right\} \\
|u \| v|^{2 / \mathbf{t}\left(\theta^{-1}\right)} & =\exp \left\{-2 \pi(\mathbf{n}(\theta) / \mathbf{t}(\theta))\left(\Im(z)+\Im\left(z^{\prime}\right)\right)\right\}
\end{aligned}
$$

obtained applying (2.8), we see that

$$
\mathcal{D} \cap \mathbb{T}=\Phi(\mathcal{W}), \quad \mathcal{D}^{+} \cap \mathbb{T}=\Phi\left(\mathcal{W}^{+}\right)
$$

Let us notice that $\underline{u}_{1}, \ldots, \underline{u}_{m}, \underline{v}$ are all points of infinite order because torsion points all lie on the set $\{(u, v)$ such that $|u|,|v|<1\}$, contained in the boundaries of $\mathcal{D}$ and $\mathcal{D}^{+}$.

Let $\left(z_{1}, z_{1}^{\prime}\right), \ldots,\left(z_{m}, z_{m}^{\prime}\right),\left(z_{0}, z_{0}^{\prime}\right) \in \mathbb{C}^{2}$ be such that $\Phi\left(z_{i}, z_{i}^{\prime}\right)=\underline{u}_{i}$ for $i=$ $1, \ldots, m$ and $\Phi\left(z_{0}, z_{0}^{\prime}\right)=\underline{v}$. After (4.11), $z_{i} \in \mathcal{H}(i=1, \ldots, m)$. Since $\underline{u}_{1}=$ $\Phi\left(\alpha_{1}, \alpha_{1}^{\prime}\right) \underline{v}^{\beta_{1}}$, we have $z_{1}=\beta_{1} z_{0}$ and we have two cases; $\beta_{1}>0$ and $\Im\left(z_{0}\right)>0$ or $\beta_{1}<0$ and $\Im\left(z_{0}\right)<0$.

If $\beta_{1}<0$ and $\Im\left(z_{0}\right)<0$ we have, from (2.7):

$$
\underline{u}_{i}=\Phi\left(\alpha_{i}, \alpha_{i}^{\prime}\right) \underline{s}^{-\beta_{i}}, \quad i=1, \ldots, m
$$


with $\underline{s}=\underline{v}^{-1}=\Phi\left(-z_{0},-z_{0}^{\prime}\right)$. By Proposition 4.5 , the series $\mathbb{F}\left(\Phi\left(\alpha_{i}, \alpha_{i}^{\prime}\right) \underline{U}^{\beta_{i}}\right)$ are $\mathbb{Q}$-linearly independent if and only if the series $\mathbb{F}\left(\Phi\left(\alpha_{i}, \alpha_{i}^{\prime}\right) \underline{U}^{-\beta_{i}}\right)$ are $\mathbb{Q}$-linearly independent $(i=1, \ldots, m)$ so that to prove Lemma 4.7, it is enough to consider the case $\beta_{1}>0$ and $\Im\left(z_{0}\right)>0$, and in this case, $\beta_{i}>0$ for all $i$.

By Lemma 4.6, $W$ is non-trivial. Let $\eta$ be an element of $W$ such that $\eta>1>$ $\eta^{\prime}>0$. There exists $k \geq 0$ such that $\eta^{k} \mathfrak{s}\left(z_{0}\right)>\eta^{\prime k}\left|\mathfrak{s}\left(z_{0}^{\prime}\right)\right|>0$; we denote by $\underline{a}$ the point $\underline{v}^{\eta^{k}}=\Phi\left(\eta^{k} z_{0}, \eta^{\prime-k} z_{0}^{\prime}\right)$. Since $\left(\eta^{k} z_{0}, \eta^{\prime k} z_{0}^{\prime}\right) \in \mathcal{W}^{+}$, we have $\underline{a} \in \mathcal{D}^{+}$by (4.11).

We also have $\underline{u}_{i}=\Phi\left(\alpha_{i}, \alpha_{i}^{\prime}\right) \underline{a}^{\eta^{-k}} \beta_{i}$ (and $\eta^{-k} \beta_{i}>0$ for all $i$ ). For the same reasons as above, there exists $\ell>0$ such that $\underline{w}_{i}:=\underline{u}_{i}^{\eta^{\ell}} \in \mathcal{D}^{+}$and $\gamma_{i}:=\eta^{\ell-k} \beta_{i}$ $(i=1, \ldots, m)$ are such that (4.9) holds.

Since $\gamma_{i} \in W \beta_{i}$ for all $i=1, \ldots, m$, Proposition 4.5 implies that the series $\mathbb{F}\left(\Phi\left(\alpha_{i}, \alpha_{i}^{\prime}\right) \underline{U}^{\beta_{i}}\right)$ are $\mathbb{Q}$-linearly independent if and only if the series $\mathbb{F}\left(\Phi\left(\alpha_{i}, \alpha_{i}^{\prime}\right) \underline{U}^{\gamma_{i}}\right)$ are $\mathbb{Q}$-linearly independent.

Proposition 4.8. Let $\mathcal{M}=\left(\underline{u}_{1}, \ldots, \underline{u}_{m}\right)$ be an $m$-tuple of algebraic elements of $\mathbb{T} \cap \mathcal{D}$ satisfying (2.7) (rank one hypothesis). Let us suppose that there exist rational numbers $c_{1}, \ldots, c_{m}$, not all zero, such that

$$
\sum_{i=1}^{m} c_{i} \mathbb{F}\left(\Phi\left(\alpha_{i}, \alpha_{i}^{\prime}\right) \underline{U}^{\beta_{i}}\right)=0
$$

Then, there exist rational numbers $d_{1}, \ldots, d_{m}$ not all zero and an algebraic number $\lambda$ such that

$$
\sum_{i=1}^{m} d_{i} f\left(\underline{u}_{i}\right)=\lambda
$$

Proof. Let us write again, as in (4.5) and (4.6), $\underline{\zeta}_{i}=\Phi\left(\alpha_{i}, \alpha_{i}^{\prime}\right), \underline{\zeta}_{i}^{\sharp}=\Phi\left(\alpha_{i} / \beta_{i}, \alpha_{i}^{\prime} / \beta_{i}^{\prime}\right)$ $(i=1, \ldots, m)$. We apply Lemma 4.7; let $\underline{w}_{1}, \ldots, \underline{w}_{m}, \underline{a}, \gamma_{1}, \ldots, \gamma_{m}$ be given by this lemma.

The $m$-tuple $\left(\underline{w}_{1}, \ldots, \underline{w}_{m}\right)$ clearly satisfies the rank one hypothesis (cf. (4.9)). Since $\gamma_{i}= \pm \eta^{s} \beta_{i}$ for some $s \in \mathbb{Z}$, Proposition 4.5 implies that

$$
\sum_{i=1}^{m} c_{i} \mathbb{F}\left(\underline{\zeta}_{i} \underline{U}^{\gamma_{i}}\right)=0
$$

With $\mathcal{F}=\mathcal{C}(]-1,0[)$, Proposition 4.4 and Proposition 3.5 point 5 imply

$$
\left.\sum_{i=1}^{m} c_{i} f_{\gamma_{i}^{-1} M}\left(\underline{\zeta}_{i}^{\sharp} \underline{U}\right)=0 \quad \text { (in } \mathbb{C}\{\underline{U}\}\right) .
$$


Let us assume, without loss of generality, that there exists $m_{0}$, with $0 \leq m_{0} \leq m$, such that:

$$
\begin{aligned}
& \gamma_{i}^{\prime}>0 \text { for } i=1, \ldots, m_{0}, \\
& \gamma_{i}^{\prime}<0 \text { for } i=m_{0}+1, \ldots, m .
\end{aligned}
$$

The existence of $m_{0}$ is guaranteed after reordering the indexes $i$ (if $m_{0}=0$ or $m_{0}=m$, one of the cases is empty). We have, by definition of $R_{\alpha, \beta}, R_{\alpha, \beta}^{+}$and by point 2 of Proposition 3.5:

$$
\begin{aligned}
0 & =\sum_{i=1}^{m} c_{i} f_{\gamma_{i}^{-1} M}\left(\underline{\zeta}_{i}^{\sharp} \underline{U}\right) \\
& =\sum_{i=1}^{m_{0}} c_{i} f_{\gamma_{i}^{-1} M}\left(\underline{\zeta}_{i}^{\sharp} \underline{U}\right)+\sum_{j=m_{0}+1}^{m} c_{j} f_{\gamma_{j}^{-1} M}\left(\underline{\zeta}_{j}^{\sharp} \underline{U}\right) \\
& \left.=\sum_{i=1}^{m_{0}} c_{i} f\left(\underline{\zeta}_{i} \underline{U}^{\gamma_{i}}\right)+\sum_{j=m_{0}+1}^{m} c_{j} f^{+}\left(\underline{\zeta}_{j} \underline{U}^{\gamma_{j}}\right)+\sum_{i=1}^{m} c_{i} R_{\alpha_{i}, \gamma_{i}} \underline{U}\right) \\
& =\sum_{i=1}^{m_{0}} c_{i} f\left(\underline{\zeta}_{i} \underline{U}^{\gamma_{i}}\right)-\sum_{j=m_{0}+1}^{m} c_{j} f\left(\underline{\zeta}_{j} \underline{U}^{\gamma_{j}}\right)+\Lambda(\underline{U}),
\end{aligned}
$$

where

$$
\Lambda(\underline{U})=\sum_{i=1}^{m} c_{i} R_{\alpha_{i}, \gamma_{i}}(\underline{U})+\sum_{j=m_{0}+1}^{m} c_{j} \Theta\left(\underline{\zeta}_{j} \underline{U}^{\gamma_{j}}\right) .
$$

Since $\gamma_{i} \geq\left|\gamma_{i}^{\prime}\right|>0$ for all $i$, Proposition 3.6 point 2 implies that the series $R_{\alpha_{i}, \gamma_{i}}(\underline{u})$ simultaneously converge for $\underline{u} \in \mathcal{D}^{+}$. By Proposition 3.5 point 6 , the series $\Theta\left(\underline{\zeta}_{j} \underline{u}^{\gamma_{j}}\right)$ also converge on $\mathcal{D}^{+}$, and $\Lambda(\underline{u})$ converge on $\mathcal{D}^{+}$. Similarly, the series $f\left(\underline{\zeta}_{i} \underline{u}^{\gamma_{i}}\right)$ converge on $\mathcal{D}^{+}$(by Proposition 3.5 point 3 ), so that (4.13) gives rise to a relation between series converging on $\mathcal{D}^{+}$(at each step, the series involved in the linear combinations converge on $\mathcal{D}^{+}$).

After Proposition 3.6 point 3 , the series $R_{\alpha_{i}, \gamma_{i}}$ simultaneously converge to rational functions of $\overline{\mathbb{Q}}(\underline{u})$. Looking directly at the definition of these functions, we also see for general $\alpha \in K, \beta \in S \backslash\{0\}$ with $\beta \geq\left|\beta^{\prime}\right|>0, \underline{\zeta}=\Phi\left(\alpha, \alpha^{\prime}\right)$ and $\underline{\zeta}^{\sharp}=\Phi\left(\alpha / \beta, \alpha^{\prime} / \beta^{\prime}\right)$, that $R_{\alpha, \beta}(\underline{U})+R_{\alpha, \beta}^{+}(\underline{U})=\Theta_{\beta^{-1} M}\left(\underline{\zeta^{\sharp}} \underline{U}\right)-\Theta\left(\underline{\zeta} \underline{U}^{\beta}\right)$ in $\mathbb{C}\{\underline{U}\}$.

Thus, for all $i$,

$$
\Theta\left(\underline{\zeta}_{i} \underline{U}^{\gamma_{i}}\right)=\Theta_{\gamma_{i}^{-1} M}\left(\underline{\zeta}_{i}^{\sharp} \underline{U}\right)-R_{\alpha_{i}, \gamma_{i}}(\underline{U})-R_{\alpha_{i}, \gamma_{i}}^{+}(\underline{U}) .
$$

By Proposition 3.5 point $4, \Theta_{\gamma_{i}^{-1} M}\left(\underline{\zeta}_{i}^{\sharp} \underline{u}\right)$ converges on $\mathcal{D}^{+}$to a rational function of $\overline{\mathbb{Q}}(\underline{u})$ (for all $i$ ). Hence, for all $i$, the same is true for $\Theta\left(\underline{\zeta}_{i} \underline{u}^{\gamma_{i}}\right)$. 
Therefore, we have proved that the series $\Lambda(\underline{u})$ converges on $\mathcal{D}^{+}$to a rational function of $\overline{\mathbb{Q}}(\underline{u})$. Since $\underline{w}_{1}, \ldots, \underline{w}_{m}, \underline{a}$ belong to $\mathcal{D}^{+}, \kappa:=\Lambda(\underline{a}) \in \overline{\mathbb{Q}}$, and by (4.13) we obtain

$$
\sum_{i=1}^{m} d_{i} f\left(\underline{w}_{i}\right)=\kappa
$$

where $d_{i}=-c_{i}$ for $i=1, \ldots, m_{0}$ and $d_{i}=c_{i}$ for $i=m_{0}+1, \ldots, m$.

By Lemma 4.7, the $\underline{w}_{i}$ 's and the $\underline{u}_{i}$ 's are related by $\underline{w}_{i}=\underline{u}_{i}^{\eta^{*}}$ for all $i$, so that after (1.11), we obtain a linear relation like (4.12).

In the rest of this article we prove the other implication of the theorem, that is: if $\underline{u}_{1}, \ldots, \underline{u}_{m} \in \mathbb{T}(\overline{\mathbb{Q}}) \cap \mathcal{D}$ satisfy the rank one hypothesis and are such that the numbers $f\left(\underline{u}_{1}\right), \ldots, f\left(\underline{u}_{m}\right)$ are algebraically dependent over $\mathbb{Q}$, then a relation like (1.5) holds. The existence of a linear relation between the numbers $f\left(\underline{u}_{1}\right), \ldots, f\left(\underline{u}_{m}\right)$ will follow from Proposition 4.8.

\section{Irrationality and linear independence}

Let us denote by $\Pi: \mathbb{R}^{2} \rightarrow \mathbb{R}$ the projection on the second coordinate.

Lemma 5.1 (A criterion of irrationality). Let $Q(\underline{U})$ be a formal series in two variables with complex coefficients, whose $K$-support is contained in $\Sigma\left(M_{+}^{*}\right)$ (respectively $\Sigma\left(M_{ \pm}^{*}\right)$ ). Let us suppose that the set $\Pi\left(\Sigma_{K}(Q)\right)$ contains a sequence of points $\left(x_{s}^{\prime}\right)_{s \in \mathbb{N}} \in K \backslash\{0\}$ such that:

$$
\lim _{s \rightarrow \infty} x_{s}^{\prime}=0
$$

and that $Q$ converges on a non-empty neighbourhood $\mathcal{O}$ of $\underline{0}$. Then, over $\mathcal{O}, Q$ does not converge to a rational function of $\mathbb{C}(\underline{u})$.

Proof. We only deal with the case of the $K$-support contained in $\Sigma\left(M_{+}^{*}\right)$; the other case allows a very similar proof, and is left to the reader. Thus

$$
Q(\underline{U})=\sum_{\nu \in M_{+}^{*}} q_{v} M(\underline{U})^{v}
$$

(notation introduced in Section 3.4) is a formal series such that if $q_{v} \neq 0$, then $v, v^{\prime}>0$. At the same time, $Q(\underline{u})$ converges on $\mathcal{O} \ni(0,0)$ so that, if $\underline{u} \in \mathcal{O}$,

$$
Q(\underline{u})=\sum_{(l, h) \in \mathbb{N}^{2}} q_{l, h} u^{l} v^{h}
$$

for complex numbers $q_{l, h}$ by hypothesis. 
Let $B$ be in $\mathbb{C}[\underline{U}]$. We may write:

$$
B(\underline{U})=\sum_{v \in \mathcal{E}} c_{\nu} M(\underline{U})^{v},
$$

where $\mathcal{E}:=\Sigma_{K}(B)$ is a non-empty finite subset of $\Sigma\left(M^{*}\right)$ such that if $v \in \mathcal{E}$, then $M(\underline{U})^{v} \in \mathbb{C}[\underline{U}]$.

Cauchy's product $(B Q)(\underline{U})$ of $B(\underline{U})$ and $Q(\underline{U})$ is well defined as a series of $\mathbb{C}\{\underline{U}\}$, because

$$
\begin{aligned}
(B Q)(\underline{U}) & =\sum_{\nu \in \mathcal{E}} c_{\nu} M(\underline{U})^{v} Q(\underline{U}) \\
& =\sum_{\nu \in \mathcal{E}} \sum_{\mu \in M_{+}^{*}} c_{\nu} q_{\mu} M(\underline{U})^{\mu+v} \\
& =\sum_{\tau \in M^{*}} p_{\tau} M(\underline{U})^{\tau}
\end{aligned}
$$

where $p_{\tau}$ is the finite sum $\sum c_{\nu} q_{\mu}$ running over the finite set $\{(\nu, \mu) \in \mathcal{E} \times$ $M_{+}^{*}$ such that $\nu+\mu=\tau$.

Moreover, by (5.2), $B Q$ converge on $\mathcal{O}$ and we have $(B Q)(\underline{u})=B(\underline{u}) Q(\underline{u})$ for all $u \in \mathcal{O}$.

We have that $Q$ converges on $\mathcal{O}$ to a rational function if and only if there exists a polynomial function $B \in \mathbb{C}[\underline{u}]$ such that, over $\mathcal{O}, B(\underline{u}) Q(\underline{u})$ is a polynomial function, and this is equivalent to the finiteness of the $K$-support of the formal series $(B Q)(\underline{U})$.

In order to prove the lemma we need to show that, under our hypotheses, for any non-zero polynomial $B \in \mathbb{C}[\underline{U}]$, the $K$-support of the series $B Q$ is infinite.

Let us introduce the number

$$
\lambda_{0}:=\left(\min _{\lambda \in \mathcal{E}} \lambda^{\prime}\right)^{\prime} \in K
$$

For all $i$ we write $v_{i}:=x_{i}+\lambda_{0}$. From (5.1) we deduce that $\lim _{i \rightarrow \infty} v_{i}^{\prime}=\lambda_{0}^{\prime}$. Let us write, for $\lambda \in M^{*}, F_{\lambda}(\underline{u})=M(\underline{u})^{\lambda} Q(\underline{u})$. We have:

$$
\Sigma\left(v_{i}\right) \in \Sigma_{K}\left(F_{\lambda_{0}}\right), \quad \text { for all } i \in \mathbb{N} .
$$

As $x_{i}^{\prime}$ becomes very close to 0 we have:

$$
\Sigma\left(v_{i}\right) \notin \bigcup_{\lambda \in \mathcal{E} \backslash\left\{\lambda_{0}\right\}} \Sigma_{K}\left(F_{\lambda}\right) .
$$

Thus, for $i$ big enough, $\Sigma\left(v_{i}\right) \in \Sigma_{K}(B Q)$, so that the $K$-support of $B Q$ is infinite (we recall that $Q$ is a series involving terms $q_{v} M(\underline{U})^{v}$ with $v^{\prime}>0$ ). 


\subsection{The main proposition}

Under certain conditions, Lemma 5.1 becomes an equivalence, thus a tool to check linear independence of functions. In this section we investigate this property. Proposition 5.2 below gives a converse of Lemma 5.1 under certain hypotheses.

We need some additional notations. Let $\alpha_{1}, \ldots, \alpha_{m}$ be elements of $K$ and $\gamma_{1}, \ldots, \gamma_{m}$ be elements of $S \backslash\{0\}$, such that $\gamma_{i} \geq\left|\gamma_{i}^{\prime}\right|>0$ for all $i=1, \ldots, m$; let us also write $N_{i}=\gamma_{i}^{-1} M$ and let us consider again the torsion points $\underline{\zeta}_{1}, \ldots, \underline{\zeta}_{m}$ defined as in (4.5). As in Section 4.2, we assume (without loss of generality) that there exists $m_{0}$, with $0 \leq m_{0} \leq m$, such that:

$$
\begin{aligned}
& \gamma_{i}^{\prime}>0 \text { for } i=1, \ldots, m_{0}, \\
& \gamma_{i}^{\prime}<0 \text { for } i=m_{0}+1, \ldots, m .
\end{aligned}
$$

We define:

$$
\begin{array}{ll}
\phi_{i}(\underline{U})=f\left(\underline{\zeta}_{i} \underline{U}^{\gamma_{i}}\right), & \text { for } i=1, \ldots, m_{0}, \\
\phi_{i}(\underline{U})=f^{+}\left(\underline{\zeta}_{i} \underline{U}^{\gamma_{i}}\right) & \text { for } i=m_{0}+1, \ldots, m .
\end{array}
$$

Since $\gamma_{i} M_{ \pm}^{*} \subset M_{ \pm}^{*}$ for $i=1, \ldots, m_{0}$ and $\gamma_{i} M_{+}^{*} \subset M_{ \pm}^{*}$ for $i=m_{0}+1, \ldots, m$, for all $i=1, \ldots, m$, we have

$$
\Sigma_{K}\left(\phi_{i}\right) \subset N_{i \pm}^{*}, \quad i=1, \ldots, m .
$$

Together with the formal series $\phi_{i}$, we also consider:

$$
\begin{aligned}
& \phi_{i}^{+}(\underline{U})=f^{+}\left(\underline{\zeta}_{i} \underline{U}^{\gamma_{i}}\right), \quad \text { for } i=1, \ldots, m_{0}, \\
& \phi_{i}^{+}(\underline{U})=f\left(\underline{\zeta}_{i} \underline{U}^{\gamma_{i}}\right), \quad \text { for } i=m_{0}+1, \ldots, m,
\end{aligned}
$$

and we check in a similar way that:

$$
\Sigma_{K}\left(\phi_{i}^{+}\right) \subset N_{i+}^{*}, \quad i=1, \ldots, m .
$$

By Proposition 3.5 point 6 , the series $\phi_{i}$ converge on $\mathcal{D}$ while the series $\phi_{i}^{+}$converge on $\mathcal{D}^{+}$(for all $i=1, \ldots, m$ ).

Let $c_{1}, \ldots, c_{m}$ be complex numbers. Let us write:

$$
\begin{gathered}
Q(\underline{U})=\sum_{i=1}^{m} c_{i} \phi_{i}(\underline{U}), \\
Q^{+}(\underline{U})=\sum_{i=1}^{m} c_{i} \phi_{i}^{+}(\underline{U}) .
\end{gathered}
$$

The series $Q$ converges on $\mathcal{D}$ and the series $Q^{+}$converges on $\mathcal{D}^{+}$. Let us write

$$
\underline{\zeta}_{i}^{\sharp}=\Phi\left(\frac{\alpha_{i}}{\gamma_{i}}, \frac{\alpha_{i}^{\prime}}{\gamma_{i}^{\prime}}\right), \quad i=1, \ldots, m,
$$

so that for all $i, \underline{\zeta}_{i}^{\sharp} \gamma_{i}=\underline{\zeta}_{i}$. 
Proposition 5.2. The following conditions are equivalent:

(1) The series $Q^{+}(\underline{u})$ in (5.4) converges on $\mathcal{D}^{+}$to a rational function.

(2) There exists a positive real number $\epsilon$ such that for all $\alpha \in \Pi\left(\Sigma_{K}\left(Q^{+}\right)\right)$, we have $\alpha>\epsilon$.

(3) We have, in $\mathbb{C}\{\underline{U}\}$ :

$$
\sum_{i=1}^{m} c_{i} f_{N_{i}}^{+}\left(\underline{\zeta}_{i}^{\sharp} \underline{U}\right)=0
$$

(4) The series $Q(\underline{u})$ in (5.3) converges on $\mathcal{D}$ to a rational function.

(5) There exists a positive real number $\omega$ such that for all $\alpha \in \Pi\left(\Sigma_{K}(Q)\right)$, we have that $\alpha<-\omega$.

(6) We have, in $\mathbb{C}\{\underline{U}\}$ :

$$
\sum_{i=1}^{m} c_{i} f_{N_{i}}\left(\underline{\zeta}_{i}^{\sharp} \underline{U}\right)=0 .
$$

Proof. We first prove that (3) implies (1); the proof is similar to part of the proof of Proposition 4.8. We have, for all $i=1, \ldots, m$, that the series

$$
R_{\alpha_{i}, \gamma_{i}}^{+}(\underline{U})=f_{N_{i}}^{+}\left(\underline{\zeta}_{i}^{\sharp} \underline{U}\right)-\phi_{i}^{+}(\underline{U})
$$

converges on $\mathcal{D}^{+}$to a rational function (use Proposition 3.6 points 2, 3). Thus

$$
Q^{+}(\underline{u})=\sum_{i=1}^{m} c_{i} \phi_{i}^{+}(\underline{u})=-\sum_{i=1}^{m} c_{i} R_{\alpha_{i}, \gamma_{i}}^{+}(\underline{u})
$$

converges to a rational function on $\mathcal{D}^{+}$.

That (1) implies (2) follows from Lemma 5.1: first of all, as $Q^{+}$converges on $\mathcal{O}:=\mathcal{D}^{+}$, which is a non-empty neighbourhood of $(0,0)$. By Proposition 3.6 point 1 , the $K$-support of $Q^{+}(\underline{U})$ is contained in $\Sigma\left(M_{+}^{*}\right)$. As $Q^{+}(\underline{u})$ converges on $\mathcal{O}$ to a rational function, 0 is not an accumulation point of $\Pi\left(\Sigma_{K}\left(Q^{+}\right)\right)$and the required positive real number $\epsilon$ exists.

We now show that (2) implies (3). By hypothesis, for every element $\tau^{\prime} \in$ $\Pi\left(\Sigma_{K}\left(Q^{+}\right)\right)$,

$$
\tau^{\prime}>\epsilon
$$

for some $\epsilon>0$. From the definition of the series $R_{\alpha, \beta}, R_{\alpha, \beta}^{+}$, we obtain the equality:

$$
\sum_{i=1}^{m} c_{i} f_{N_{i}}^{+}\left(\underline{\zeta}_{i}^{\sharp} \underline{U}\right)=Q^{+}(\underline{U})+R(\underline{U}),
$$

where

$$
R(\underline{U})=\sum_{i=1}^{m} c_{i} R_{\alpha_{i}, \gamma_{i}}^{+}(\underline{U})
$$


converges on $\mathcal{D}^{+}$to a rational function, (use Proposition 3.6 points 2, 3). Point 1 of Proposition 3.6 ensures us that the $K$-support of $R$ lies inside $\Sigma\left(M_{+}^{*}\right)$. By Lemma 5.1 applied to $R$ (which converges in a neighbourhood of $(0,0)$ and has its $K$-support in $\left.\Sigma\left(M_{+}^{*}\right)\right)$, there exists $\epsilon^{\prime}>0$ such that for all $\tau^{\prime} \in \Pi\left(\Sigma_{K}(R)\right)$,

$$
\tau^{\prime}>\epsilon^{\prime}
$$

Let $\mathcal{Q}$ be the $K$-support of $Q^{+}+R$. We obtain from above, that for all $\tau^{\prime} \in \Pi(\mathcal{Q})$,

$$
\tau^{\prime}>\epsilon^{\prime \prime},
$$

with $\epsilon^{\prime \prime}=\min \left(\epsilon, \epsilon^{\prime}\right)$.

Let $\eta>1>\eta^{\prime}>0$ be a unit satisfying

$$
\underline{\zeta}_{i}^{\eta}=\underline{\zeta}_{i}, \quad i=1, \ldots, m,
$$

whose existence is ensured by Lemma 4.6. Let $\mathcal{P}$ be the $K$-support of the series

$$
P(\underline{U})=\sum_{i=1}^{m} c_{i} R_{\eta, N_{i}}^{+}\left(\underline{\zeta}_{i}^{\sharp} \underline{U}\right) .
$$

We first claim that

$$
\mathcal{Q}=\bigsqcup_{k=0}^{\infty} \eta^{k} \mathcal{P}
$$

where for $\kappa \in K, \kappa \mathcal{P}$ denotes the set

$$
\left\{\left(\kappa \nu, \kappa^{\prime} \nu^{\prime}\right) \text { such that } \Sigma(v) \in \mathcal{P}\right\} .
$$

From (3.13) we see that for all $i=1, \ldots, m$ :

$$
f_{N_{i}}^{+}\left(\underline{\zeta}_{i}^{\sharp} \underline{U}\right)=\sum_{k=0}^{\infty} R_{\eta, N_{i}}^{+}\left(\left(\underline{\zeta}_{i}^{\sharp} \underline{U}\right)^{\eta^{k}}\right) .
$$

Now, if $v \in N_{i}^{*}$, then $v=\gamma_{i} \mu$ for some $\mu \in M^{*}$. By (5.5), $\underline{\zeta}_{i}^{\sharp \gamma_{i}}=\underline{\zeta}_{i}$ and applying (5.9), we get:

$$
M\left(\left(\underline{\zeta}_{i}^{\sharp}\right)^{\eta^{k}}\right)^{v}=M\left(\underline{\zeta}_{i}^{\sharp \gamma_{i}} \eta^{k}\right)^{\mu}=M\left(\underline{\zeta}_{i}\right)^{\mu}=M\left(\underline{\zeta}_{i}^{\sharp}\right)^{v},
$$

which implies

$$
M\left(\left(\underline{\zeta}_{i}^{\sharp} \underline{U}\right)^{\eta^{k}}\right)^{\nu}=M\left(\underline{\zeta}_{i}^{\sharp \eta^{k}}\right)^{v} M\left(\underline{U}^{\eta^{k}}\right)^{v}=M\left(\underline{\zeta}_{i}^{\sharp} \underline{U}^{\eta^{k}}\right)^{\nu} .
$$

Thus we have, for all $k$,

$$
R_{\eta, N_{i}}^{+}\left(\left(\underline{\zeta}_{i}^{\sharp} \underline{U}^{\eta^{k}}\right)=R_{\eta, N_{i}}^{+}\left(\underline{\zeta}_{i}^{\sharp} \underline{U}^{\eta^{k}}\right),\right.
$$


which implies

$$
f_{N_{i}}^{+}\left(\underline{\zeta}_{i}^{\sharp} \underline{U}\right)=\sum_{k=0}^{\infty} R_{\eta, N_{i}}^{+}\left(\underline{\zeta}_{i}^{\sharp} \underline{U}^{\eta^{k}}\right) .
$$

From (5.8) and (5.11):

$$
\begin{aligned}
Q^{+}(\underline{U})+R(\underline{U}) & =\sum_{i=1}^{m} c_{i} f_{N_{i}}^{+}\left(\underline{\zeta}_{i}^{\sharp} \underline{U}\right) \\
& =\sum_{i=1}^{m} c_{i} \sum_{k=0}^{\infty} R_{\eta, N_{i}}^{+}\left(\underline{\zeta}_{i}^{\sharp} \underline{U}^{\eta^{k}}\right) \\
& =\sum_{k=0}^{\infty} P\left(\underline{U}^{\eta^{k}}\right),
\end{aligned}
$$

so that $\mathcal{Q} \subseteq \cup_{k=0}^{\infty} \eta^{k} \mathcal{P}$. From (3.14), $\mathcal{P} \subset \Sigma\left(\mathcal{D}_{+}(\eta)\right)$ so that if $k \neq h, \eta^{k} \mathcal{P} \cap \eta^{h} \mathcal{P}=$ $\emptyset, \mathcal{Q} \supseteq \sqcup_{k=0}^{\infty} \eta^{k} \mathcal{P}$ and the claim follows.

We want to prove that $\mathcal{P}$ is empty; this will imply that $\mathcal{Q}$ is empty too, by the claim. Let us suppose by contradiction that there exists some $v$ such that $\Sigma(v) \in \mathcal{P}$. Then for all $k \geq 0$ we have that $\Sigma\left(\eta^{k} v\right) \in \mathcal{Q}$ by (5.10). As

$$
\lim _{k \rightarrow \infty}\left(\eta^{k} v\right)^{\prime}=0
$$

we see that for some $k$ big enough

$$
\epsilon^{\prime \prime}>\left(\eta^{k} v\right)^{\prime}>0,
$$

which implies that there exists some element $\tau$ with $\Sigma(\tau) \in \mathcal{Q}$ such that $0<\tau^{\prime}<$ $\epsilon^{\prime \prime}$ : this gives us the required contradiction.

Thus, $\mathcal{P}=\mathcal{Q}=\emptyset$. But $\mathcal{Q}$ is also the $K$-support of the left hand side of (5.8), which vanishes. Assuming part (2) of the proposition, it is now clear that (5.6) holds.

Let us prove that (1) implies (4). The point 4 of Proposition 3.5 implies that, on $\mathcal{D}^{+}$,

$$
\phi_{i}(\underline{u})=-\phi_{i}^{+}(\underline{u})+R_{i}(\underline{u})
$$

for rational functions $R_{i}(\underline{u}) \in \overline{\mathbb{Q}}(\underline{u})$ and for all $i=1, \ldots, m$. Thus, on $\mathcal{D}^{+}$

$$
Q(\underline{u})=-Q^{+}(\underline{u})+\sum_{i=1}^{m} c_{i} R_{i}(\underline{u})
$$

and $Q(\underline{u})$ converges to a rational function on $\mathcal{D}^{+}$. But $Q(\underline{u})$ also converges on $\mathcal{D}$; thus it converges to a rational function on $\mathcal{D}$.

The proof that (4) implies (1) is essentially the same, due to the fact that $\mathcal{D}^{+} \subset \mathcal{D}$. 
The proof that (4), (5), (6) are equivalent, runs along the same ideas than the proof for the equivalence of (1), (2), (3). It is enough to notice that by Proposition 3.6 point 1, the series $Q(\underline{U})$ has its $K$-support contained in $\Sigma\left(M_{ \pm}^{*}\right)$.

\section{End of proof of the theorem}

In this section we quote a criterion of algebraic independence of Nishioka and we conclude the proof of our theorem.

\subsection{Mahler's theory}

As noticed earlier, the set $\mathcal{D}^{+}$is a non-empty open neighbourhood of $\underline{0}$. Let $\eta$ be a unit of $S$ such that $\eta>1>\eta^{\prime}>0$. It is easy to check that the matrix $\mathcal{B}(\eta)$ has non-negative integer entries, and eigenvalues $\eta, \eta^{\prime}$. For $\underline{a} \in \mathcal{D}^{+}$an algebraic point of $\mathbb{T}$, it follows that the couple $(\Omega, \alpha)$, with $\Omega=\mathcal{B}(\eta)$ and $\alpha=a$, satisfies the properties I, II, III page 33 of [Ni]. In particular $\mathcal{B}(\eta)^{k} . \underline{a} \in \mathcal{D}^{+}$for all $k \in \mathbb{N}$ and

$$
\lim _{k \rightarrow \infty} \mathcal{B}(\eta)^{k} \cdot \underline{a}=\underline{0} \text {. }
$$

By using Mahler's vanishing theorem (cf. [Ni, Theorem 2.2, page 36], or [Mah]), we also check the property IV page 34 of [Ni] (see also page 45).

Let $\Psi_{1}(u), \ldots, \Psi_{m}(\underline{u})$ be functions in two variables, analytic on $\mathcal{D}^{+}$, and let $\underline{a} \in \mathcal{D}^{+}$be an algebraic point of $\mathbb{T}$. Let us suppose that the functions $\Psi_{i}$ satisfy on $\mathcal{D}^{+}$a system of functional equations:

$$
\Psi_{i}(\mathcal{B}(\eta) . \underline{u})=\Psi_{i}(\underline{u})+R_{i}(\underline{u}) \text { for } 1 \leq i \leq m,
$$

where $R_{1}, \ldots, R_{m}$ are rational functions, defined on $\mathcal{D}^{+}$. Let us also suppose that the Taylor series at $\underline{0}$ of $\Psi_{i}$ and the coefficients of $R_{i}$ are algebraic numbers in a given number field, for all $i=1, \ldots, m$.

The following proposition is a direct consequence of [Ni, Theorem 3.3.2, page 88].

Proposition 6.1. If

$$
\Psi_{1}(\underline{u}), \ldots, \Psi_{m}(\underline{u})
$$

are algebraically independent over $\mathbb{C}(\underline{u})$, then the complex numbers

$$
\Psi_{1}(\underline{a}), \ldots, \Psi_{m}(\underline{a})
$$

are algebraically independent over $\mathbb{Q}$.

This proposition also follows directly from the criterion of algebraic independence of Loxton and van der Poorten page 399 of [Lo-Po2], by noticing that the matrix $\mathcal{B}(\eta)$ is good in the sense of [Mas2], page 209, because by Mahler's vanishing theorem, the point $\underline{a}$ satisfies the property $A$ on page 398 of [Lo-Po2]. 


\subsection{Conclusion}

By Lemma 4.7 and the functional equations (1.11), it suffices to prove our theorem for algebraic points $\underline{w}_{1}, \ldots, \underline{w}_{m}, \underline{a} \in \mathbb{T} \cap \mathcal{D}^{+}$satisfying (4.9) (with $\alpha_{1}, \ldots, \alpha_{m} \in K$ and $\gamma_{1}, \ldots, \gamma_{m} \in S$ ).

Let us suppose that the series

$$
\mathbb{F}\left(\Phi\left(\alpha_{i}, \alpha_{i}^{\prime}\right) \underline{U}^{\gamma_{i}}\right), \quad i=1, \ldots, m
$$

are $\mathbb{Q}$-linearly independent, and let us suppose by contradiction that the complex numbers $f\left(\underline{w}_{1}\right), \ldots, f\left(\underline{w}_{m}\right)$ are algebraically dependent over $\mathbb{Q}$.

In view of an application of Proposition 6.1, we must exhibit a certain choice of functions $\Psi_{i}$ analytic in a neighbourhood of $\underline{0}$ for $i=1, \ldots, m$.

Following the notations of Section 5.1, we consider the functions $\Psi_{i}=\phi_{i}$ $(i=1, \ldots, m)$; they are all analytic on $\mathcal{D}^{+}$thus at $\underline{0}$ as well as at $\mathcal{B}(\eta)^{k} . \underline{a}$ for all $k \in \mathbb{N}$, where $\eta$ is a unit of $S$ such that $\eta>1>\eta^{\prime}>0$ and satisfying (5.9). Moreover, their Taylor expansions at $\underline{0}$ are defined over $\mathcal{K}=\mathbb{Q}\left(\underline{\zeta}_{1}, \ldots, \underline{\zeta}_{m}\right)$.

After (3.9) we have, on $\mathcal{D}$, for $i=1, \ldots, m_{0}$ :

$$
\begin{aligned}
\phi_{i}\left(\underline{u}^{\eta}\right) & =f\left(\underline{\zeta}_{i} \underline{u}^{\gamma_{i} \eta}\right) \\
& =f\left(\underline{\zeta}_{i}^{\eta} \underline{u}^{\gamma_{i} \eta}\right) \\
& =\phi_{i}(\underline{u})-R_{\eta}\left(\underline{\zeta}_{i} \underline{u}^{\gamma_{i}}\right) .
\end{aligned}
$$

Similarly, after (3.10) we have, on $\mathcal{D}^{+}$, for $i=m_{0}+1, \ldots, m$ :

$$
\phi_{i}\left(\underline{u}^{\eta}\right)=\phi_{i}(\underline{u})-R_{\eta}^{+}\left(\underline{\zeta}_{i} \underline{u}^{\gamma_{i}}\right) .
$$

Hence, the functions $\Psi_{i}=\phi_{i}(i=1, \ldots, m)$ satisfy on $\mathcal{D}^{+}$the collection of simultaneous functional equations (6.1), where

$$
\begin{aligned}
R_{i}(\underline{u})=-R_{\eta}\left(\underline{\zeta}_{i} \underline{u}^{\gamma_{i}}\right) \quad \text { for } i=1, \ldots, m_{0}, \\
=-R_{\eta}^{+}\left(\underline{\zeta}_{i} \underline{u}^{\gamma_{i}}\right) \quad \text { for } i=m_{0}+1, \ldots, m .
\end{aligned}
$$

After Proposition 3.6 points $2,3, R_{i}(\underline{u})$ is a rational function of $\overline{\mathbb{Q}}(\underline{u})$ which is well defined on $\mathcal{D}^{+}$for all $i$. Moreover, for all $i, R_{i}(\underline{u}) \in \mathcal{K}(\underline{u})$.

Therefore, Proposition 6.1 can be applied and says that the functions $\phi_{i}(\underline{u})$ are algebraically dependent over $\mathbb{C}(\underline{u})$.

Theorem 3.2.2 of [Ni, page 85] (see also [Ku, Corollary 9, page 29]) implies that the functions $\phi_{i}(\underline{u})$ are $\mathbb{C}$-linearly dependent modulo $\mathbb{C}(\underline{u})$ in the following sense. There exist $m$ complex numbers $c_{1}, \ldots, c_{m}$, not all zero, and a rational function $Q(\underline{u}) \in \mathbb{C}(\underline{u})$ such that:

$$
\sum_{i=1}^{m} c_{i} \phi_{i}(\underline{u})=Q(\underline{u}) .
$$


This is condition (4) of Proposition 5.2. By Proposition 5.2, we have the $\mathbb{C}$-linear relations (5.6) (and (5.7)) and, applying Proposition 4.4 with $\mathcal{F}=\mathcal{C}$ (]0, 1[) (or with $\mathcal{F}=\mathcal{C}(] 0,1[)$ if we want to use $(5.7))$, we obtain a contradiction. Thus, the formal series in (6.2) are $\mathbb{Q}$-linearly dependent, hence completing the proof of our theorem.

\section{Proof of Corollaries 1.6, 1.7}

We first consider Corollary 1.6; its proof follows closely the Example 1.3 of Section 1 concerning the relation (1.8). We go back to our notations $f_{w}, f_{\theta}$ for this proof.

Let us choose a numbering of the points $\left(\zeta^{i}, \zeta^{j}\right)$ by writing

$$
\left\{\underline{\zeta}_{1}, \ldots, \underline{\zeta}_{p^{2}}\right\}=\left\{\left(\zeta^{i}, \zeta^{j}\right), 0 \leq i, j \leq p-1\right\}
$$

Let us write $\underline{u}=(u, v)$ and $\underline{v}=T$. $\underline{u}$, where $T$ is defined as in (1.7); notice that $\underline{v}$ is certainly not a torsion point because otherwise we would have $\underline{1}=(T \cdot \underline{u})^{s}=T .\left(\underline{u}^{s}\right)$ for some integer $s>0$, and $\underline{u}$ would be torsion too (we have that $T \in \mathrm{SL}_{2}(\mathbb{Z})$ ). It is clear that $f_{\theta}$ converges at $\underline{v}$.

Let us also write $\underline{u}_{i}=\underline{\zeta}_{i} \underline{u}\left(1 \leq i \leq p^{2}\right)$. Since $\underline{\zeta}_{i}^{p}=\underline{1}$ for all $i$, we have $\underline{1}=T \cdot\left(\underline{\zeta}_{i}^{p}\right)=T \cdot\left(\left(\begin{array}{ll}p & 0 \\ 0 & p\end{array}\right) \cdot \underline{\zeta}_{i}\right)=\left(T \cdot \underline{\zeta}_{i}\right)^{p}$, which means that for all $i, T . \underline{\zeta}_{i}$ is a torsion point of order $p$. But $T \in \mathrm{SL}_{2}(\mathbb{Z})$, thus there exists a permutation $\sigma$ : $\left\{1, \ldots, p^{2}\right\} \rightarrow\left\{1, \ldots, p^{2}\right\}$ such that $T \cdot \underline{\zeta}_{i}=\underline{\zeta}_{\sigma(i)}$, that is

$$
T \cdot \underline{u}_{i}=\underline{\zeta}_{\sigma(i)} \underline{v} .
$$

By Lemma 2.1 there exist $\alpha_{1}, \ldots, \alpha_{p^{2}} \in K$ such that $\Phi\left(\alpha_{i}, \alpha_{i}^{\prime}\right)=\underline{\zeta}_{\sigma(i)}$ for all $i$, and $\left(T \cdot \underline{u}_{1}, \ldots, T \cdot \underline{u}_{p^{2}}\right)$ satisfy the rank one hypothesis.

Clearly, $f_{\theta}$ converges at $T . \underline{u}_{i}$ for all $i$. We have that $f_{w}\left(\underline{u}_{1}\right), \ldots, f_{w}\left(\underline{u}_{p^{2}}\right)$ are algebraically independent if and only if $f_{\theta}\left(T \cdot \underline{u}_{1}\right), \ldots, f_{\theta}\left(T \cdot \underline{u}_{p^{2}}\right)$ are algebraically independent. To check the latter property it is enough, by our theorem, to verify that the formal double Laurent series $\mathbb{F}\left(\underline{\zeta}_{i} \underline{U}\right)$ are $\mathbb{Q}$-linearly independent.

We apply Lemma 4.3 with $\mathcal{F}=\mathbb{R}^{2} \backslash\{\underline{0}\}$. We can take $g_{1}=\cdots=g_{m}=1$ and $\ell_{1}=\cdots=\ell_{m}=p$ so that in (4.3), $L=p$.

Let $\mathcal{N}(p)$ be defined as in the lemma. Then, the vector space $\mathcal{V}$ spanned by the $p^{2}$-tuples $\left(c_{1}, \ldots, c_{p^{2}}\right) \in \mathbb{C}^{p^{2}}$ such that

$$
c_{1} \mathbb{F}\left(\underline{\zeta}_{1}\right)+\cdots+c_{p^{2}} \mathbb{F}\left(\underline{\zeta}_{p^{2}}\right)=0
$$

is the kernel of the left multiplication by the matrix $\mathcal{M}(p) \cdot \mathcal{N}(p)$.

By (4.8), $\mathcal{M}(p) \cdot \mathcal{N}(p)$ is, after reordering rows and columns, a non-zero multiple of the identity matrix. Therefore, $\mathcal{V}=(\underline{0})$ and the series $\mathbb{F}\left(\underline{\zeta}_{i} \underline{U}\right)$ are $\mathbb{Q}$ linearly independent. The linear dependence relation (1.13) is easy to check. 
Let us prove Corollary 1.7. The rank one hypothesis is clearly satisfied. Since for all $i$, there exists

$$
v_{i} \in \beta_{i} M^{*} \backslash \bigcup_{j \neq i} \beta_{j} M^{*},
$$

the series $\mathbb{F}\left(\underline{U}^{\beta_{i}}\right)(i=1, \ldots, m)$ are $\mathbb{C}$-linearly independent, and Corollary 1.7 follows from the theorem.

\section{References}

[BS] Z. Borevich and I. Shafarevich, "Number theory", Academic Press, New York, London, 1966.

[Ku] K. K. KuвотA, On the Algebraic Independence of Holomorphic Solutions of Certain Functional Equations and their Values, Math. Ann. 227 (1977), 9-50.

[Lo-Po2] J. H. LOXTON and A. J. VAN DER POORTEN, Arithmetic properties of certain functions in several variables II, J. Australian Math. Soc. A24 (1977), 393-408.

[Lo-Po3] J. H. LOXTON and A. J. VAN DER POORTEN, Algebraic independence properties of the Fredholm series, J. Australian Math. Soc. A29 (1978), 31-45.

[Mah] K. MAHLER, Arithmetische Eigenschaften der Lösungen einer Klasse von Funktionalgleichungen, Math. Ann. 101 (1929), 342-366.

[Mas1] D. W. MASSER, A Vanishing theorem for Power Series, Invent. Math. 67 (1982), 275296.

[Mas2] D. W. MASSER Algebraic independence properties of the Hecke-Mahler series, Quart. J. Math. Oxford 50 (1999), 207-230.

[Ni] K. NishiokA, "Mahler functions and transcendence", Springer Lecture Notes in Mathematics, Vol. 1631, 1996.

[Pel1] F. Pellarin, Propriétés d'indépendance algébrique de valeurs de séries de HeckeMahler, C. R. Acad. Sci. Paris, Ser. I. 340 (2005), 861-866.

[Pel2] F. PELlaRIn, On the arithmetic properties of complex values of Hecke-Mahler series II, preprint.

[Per] O. Perron, "Die Lehre von den Kettenbrüchen", Chelsea Publishing Company, New York, 1957.

L.M.N.O., Université de Caen Campus II - Boulevard Maréchal Juin BP 5186 - F14032 Caen Cedex, France pellarin@math.unicaen.fr 